\title{
Improved Reduced-Order Fault Detection Filter Design for Polytopic Uncertain Discrete-Time Markovian Jump Systems with Time-Varying Delays
}

\author{
Lihong Rong, ${ }^{1,2}$ Xiuyan Peng $\left(\mathbb{D},{ }^{1}\right.$ Liangliang Liu, ${ }^{1}$ and Biao Zhang ${ }^{1}$ \\ ${ }^{1}$ College of Automation, Harbin Engineering University, Harbin 150001, China \\ ${ }^{2}$ College of Electrical and Information, Heilongjiang Bayi Agricultural University, Daqing 163319, China \\ Correspondence should be addressed to Xiuyan Peng; pxygll@sina.com
}

Received 5 July 2017; Revised 22 February 2018; Accepted 7 March 2018; Published 23 April 2018

Academic Editor: Carlos Gershenson

Copyright (C) 2018 Lihong Rong et al. This is an open access article distributed under the Creative Commons Attribution License, which permits unrestricted use, distribution, and reproduction in any medium, provided the original work is properly cited.

\begin{abstract}
The fault detection (FD) reduced-order filtering problem is investigated for a family of polytopic uncertain discrete-time Markovian jump linear systems (MJLSs) with time-varying delays. Under meeting the control precision requirements of the complex systems, the reduced-order fault detection filter can improve the efficiency of the fault detection. Then, by the aid of the Markovian Lyapunov function and convex polyhedron techniques, some novel time-varying delays and polytopic uncertain sufficient conditions in terms of linear matrix inequality (LMI) are proposed to insure the existence of the FD reduced-order filter. Finally, an illustrative example is provided to verify the usefulness of the given method.
\end{abstract}

\section{Introduction}

The past decades have witnessed a boom of advanced studies on theories and applications of Markov jump systems in many fields, such as networks communication systems, automotive systems, energy systems, biological systems, cyber-physical systems, aerospace systems, manufacturing, automation, smart grids, vehicular networking and connected vehicles, power systems, robotics, economic systems, and social systems [1-4]. MJLSs can effectively model dynamic hybrid systems involving stochastic switching (generally autonomous) subject to the Markov chains. For nonlinear systems, according to the characteristics of local linearization, the characteristics of nonlinear systems can be described by using multiple linear Markovian jump systems. Many fruitful results have been extended to MJLSs, such as the filtering, and estimation problem was studied in [5], state feedback control problems were considered in $[6,7]$, model reduction was presented in [8] for MJLSs with polytopic uncertainties, output feedback control problem was investigated in [9], stability and stabilization problems were addressed in [10], fault detection and diagnosis of MJLSs were studied in [11], and diverse control methodologies were considered in [12-14]. However, as an important factor governing the behaviors of MJLSs, the transition probabilities (TPs) are usually deemed to be certain and completely known, which do not change over time. In practice, the unrealistic and incomplete TPs are often encountered, especially when the accurate transition probability information takes time and resources consumption to obtain. Furthermore, the polytopic uncertain TPs are common in many engineering due to the influence of various environmental factors. Therefore, there are more rational and general MJLSs with the polytopic uncertain TRs. But there are few research literature about fault detection of Markovian jump systems with the exactly known, partially unknown, and uncertain TRs concurrently, and the loss of sensor or actuator information can be efficiently modeled by means of Markov chain frameworks. This is the need to solve the main problem, which is one of the motivations for our research.

On another active research front line, the fact that the fault detection, isolation, and fault-tolerant control techniques have affected the development of modern society is very prominent for the sake of higher safety and reliability (see [15-18]). The plant operation should be monitored in real time. When the components or instrument fault had been found, through the fault-tolerant control approach, 
the stable system performance has always be maintained. Therefore, many fault detection methodologies have been addressed in engineering applications, since failure may result in disastrous consequences and hazards for personnel, plant, and environment (see, e.g., [19-22]). For instance, many fruitful results to detect fault of systems without timevarying delays have been reported. Nevertheless, time-delays occur frequently in practical application because of the signal transmission, and they affect the system performance and even degrade the stability (see [23-26]). Many literature on the fault detection and filter design for the time-varying delays systems has been reported so far, but, up to now, due to the technical difficulty, there is no corresponding fault detection filter design result for polytopic uncertain discretetime Markovian Jump Systems with time-varying delays, which has important research value in practical application.

From what has been discussed above, an improved design method of reduced-order fault detection filter for polytopic uncertain discrete MJLSs with time-varying delays is firstly proposed. Then, the new method mentioned above is researched by using the Markovian Lyapunov functional method and Wirtinger-based inequality techniques, which convert the filtering design into a convex optimisation problem. Under meeting the control precision requirements of the complex systems, the fault detection reduced-order filter can improve the efficiency of the fault detection. Some novel significant results have been reported. The main contribution of the work in this paper is threefold. (i) The fault detection filtering problem for a class of discrete-time MJLSs with time-varying delays is extended to polytopic-type uncertain transition information for the first time. The more general and practicable results are derived. (ii) The new designed FD filtering stochastic stability condition for a kind of discretetime MJLSs with time-varying delays and polytopic uncertain TPs has been established for the first time. (iii) By applying Wirtinger-based inequality, it has shown the effectiveness of the proposed design approach, which can improve the sensitivity of fault detection and reduce the fault detection rate of false positives.

Notations. Throughout this paper, for real symmetric matrix $P, P>0$ means that $P$ is positive definite and $*$ represents the symmetric element. $\mathbb{R}^{n}$ denotes the $n$-dimensional Euclidean space, $\mathbb{R}^{m \times n}$ denotes the set of all $m \times n$ real matrices, and $N$ represents a positive integer. $\|\cdot\|$ denotes the Euclidean norm for vectors, $l_{2}[0, \infty)$ represents the space of square integrable vector functions over $\phi=\{\phi(k)\} \in l_{2}[0, \infty)$, and its norm is given by $\|\phi\|_{2}=\sqrt{E\left[\sum_{k=0}^{\infty}\|\phi\|^{2}\right]}$. $E[\cdot]$ stands for the mathematical expectation. $d_{1}$ and $d_{2}$ denote the lower and upper bounds of $d(k) . d_{12}$ is the shorthand notation for $d_{2}-d_{1} . d(k)$ is believed an interval-like time-varying delay.

\section{Problem Formulation}

In this section, we will consider a class of discrete MJLS with time-varying delays of the form,

$$
\begin{aligned}
x(k+1)= & A(\varsigma(k)) x(k)+A_{d}(\varsigma(k)) x(k-d(k)) \\
& +B(\varsigma(k)) u(k)+E(\varsigma(k)) \omega(k)
\end{aligned}
$$

$$
\begin{aligned}
& +F(\varsigma(k)) f(k), \\
y(k)= & C(\varsigma(k)) x(k)+C_{d}(\varsigma(k)) x(k-d(k)) \\
& +D(\varsigma(k)) \omega(k)+H(\varsigma(k)) f(k),
\end{aligned}
$$

where $u(k) \in \mathbb{R}^{n_{u}}$ stands for the known control input, $y(k) \in$ $\mathbb{R}^{n_{y}}$ represents the measured output, $x(k) \in \mathbb{R}^{n_{x}}$ refers to the plant state, $f(k) \in \mathbb{R}^{n_{f}}$ denotes the fault signal to be detected, $\omega(k) \in \mathbb{R}^{n_{d}}$ is the unknown disturbance signal, $u(k), \omega(k)$, and $f(k)$ are considered to belong to $l_{2}[0, \infty)$. $d(k)$ represents a time-varying delay satisfying the following assumption:

$$
1 \leq d_{1} \leq d(k) \leq d_{2},
$$

where $d_{1}, d_{2}$ denote the lower and upper bounds of $d(k)$. For this case, $d(k)$ is believed an interval-like time-varying delay. $\{\varsigma(k), k \geq 0\}$ represents a discrete-time Markovian process with values in a given finite set $S=\{1,2, \ldots, n\}$ and transition rates (TRs) for the process are defined as

$$
\begin{aligned}
& \operatorname{Prob}\{\varsigma(k+1)=j \mid \varsigma(k)=g\}=\lambda_{g j}, \\
& \forall g, j \in S, \lambda_{g j} \geq 0, \sum_{j=1}^{s} \lambda_{g j}=1 .
\end{aligned}
$$

Moreover, the TRs of the Markov process usually involve polytopic uncertain and partly unknown TRs, and these transition probability matrices $\Lambda=\left\{\lambda_{g j}\right\}$ are contained in the polytopic-type convex body $P_{\Lambda}$; that is, the mathematical expression is as follows:

$$
P_{\Lambda}:=\left\{\Lambda \mid \Lambda=\sum_{s=1}^{M} \alpha_{s} \Lambda_{s} ; \alpha_{s} \geq 0, \sum_{s=1}^{M} \alpha_{s}=1\right\},
$$

where vertices $\Lambda_{s}=\left[\lambda_{g j}\right]_{N \times N}, g, j \in S, S=1,2, \ldots, M$ contain some unknown and uncertain factors. For example, for system (1) with four variation modes, the TRM is expressed as

$$
\left[\begin{array}{llll}
\lambda_{11} & \hat{\lambda}_{12} & \tilde{\lambda}_{13} & \lambda_{14} \\
\tilde{\lambda}_{21} & \lambda_{22} & \hat{\lambda}_{23} & \lambda_{24} \\
\lambda_{31} & \lambda_{32} & \tilde{\lambda}_{33} & \hat{\lambda}_{34} \\
\tilde{\lambda}_{41} & \lambda_{42} & \lambda_{43} & \tilde{\lambda}_{44}
\end{array}\right],
$$

where unknown TRs and the polytopic uncertainties TRs are represented as the superscripts labeled with " " and " "” separately. We denote $S=S_{k}^{(g)} \cup S_{u c}^{(g)} \cup S_{u k}^{(g)}$ as follows:

$$
\begin{aligned}
& S_{k}^{(g)}:=\left\{j: \lambda_{g j} \text { is known }\right\}, \\
& S_{u c}^{(g)}:=\left\{j: \tilde{\lambda}_{g j} \text { is uncertain }\right\}, \\
& S_{u k}^{(g)}:=\left\{j: \hat{\lambda}_{g j} \text { is unknown }\right\} .
\end{aligned}
$$

Also, we define $\lambda_{u k}^{(g v)}:=\sum_{j \in S_{u k}^{(g)}} \hat{\lambda}_{g j}=1-\sum_{j \in S_{k}^{(g)}} \lambda_{g j}-$ $\sum_{j \in S_{u c}^{(g)}} \tilde{\lambda}_{g j}^{(g v)}$. 
Remark 1. Before proceeding further, it is worth briefly describing for each possible $\varsigma(k)=g, g \in S$, the matrices $A(k, \varsigma(k))=A_{g}(k), B(\varsigma(k))=B_{g}, A_{F}(\varsigma(k))=A_{F g}$, and so on for notational simplicity.

Remark 2. The transition probabilities considered in this paper are more common to the MJLSs, because the TRM includes some polytopic uncertain transition probabilities in the MJLSs with time-varying delays. Then, our important task is to design fault detection filter for the required system, where the desired structure is considered to be

$$
\begin{aligned}
\widehat{x}(k+1) & =A_{F g} \widehat{x}(k)+B_{F g} y(k), \\
\widehat{r}(k) & =C_{F g} \widehat{x}(k)+D_{F g} y(k),
\end{aligned}
$$

where $\widehat{x}(k) \in \mathbb{R}^{n_{r}}, 1 \leq n_{r} \leq n_{x}$. When $n_{r}=n_{x}, \widehat{x}(k)$ is the state variable of full-order fault detection filter; when $1 \leq n_{r}<$ $n_{x}, \widehat{x}(k)$ is the state variable of reduced-order fault detection filter. $\widehat{r}(k) \in \mathbb{R}^{n_{f}}$ is the so-called residual, and $A_{F g}, B_{F g}, C_{F g}$, and $D_{F g}$ are the filter matrices to be calculated.

In order to improve the performance of the fault detection system, we introduced the weighting fault signal $\bar{f}(k)$ which satisfies $\bar{f}(z)=W_{f}(z) f(z)$, where the matrix $W_{f}(z)$ presents a given stable weighting function matrix. $\bar{f}(z)$ and $f(z)$ denote Laplace transforms of $\bar{f}(k)$ and $f(k)$, respectively. One minimal state space realization of $\bar{f}(k)$ and $f(k)$ can be

$$
\begin{aligned}
x_{f}(k+1) & =A_{w f} x_{f}(k)+B_{w f} f(k), \\
\bar{f}(k) & =C_{w f} x_{f}(k)+D_{w f} f(k),
\end{aligned}
$$

where $x_{k}(t) \in R^{q}$ is the state vector, and $A_{w f}, B_{w f}, C_{w f}$, and $D_{w f}$ are constant matrices.

Define the weighted fault estimation error $e(k):=r_{F}(k)-$ $\bar{f}(k)$ and the state vector $\tilde{x}(k):=\left[\begin{array}{llll}x^{T}(k) & x_{F}{ }^{T}(k) & x_{f}{ }^{T}(k)\end{array}\right]^{T}$. Then, by augmenting (1) and (7), the error augmented system is obtained as follows:

$$
\begin{aligned}
\widetilde{x}(k+1) & =\widetilde{A}_{g} \widetilde{x}(k)+\widetilde{A}_{d g} E \tilde{x}(k-d(k))+\widetilde{B}_{g} \psi(k), \\
e(k) & =\widetilde{C}_{g} \widetilde{x}(k)+\widetilde{C}_{d g} E \tilde{x}(k-d(k))+\widetilde{D}_{g} \psi(k),
\end{aligned}
$$

$$
\begin{aligned}
& \text { where } \psi(k)= {\left[\begin{array}{lll}
u^{T}(k) & \omega^{T}(k) & f^{T}(k)
\end{array}\right]^{T} \text { and } } \\
& \widetilde{A}_{g}=\left[\begin{array}{ccc}
A_{g} & 0 & 0 \\
B_{F g} C_{g} & A_{F g} & 0 \\
0 & 0 & A_{w f g}
\end{array}\right], \\
& \widetilde{A}_{d g}=\left[\begin{array}{c}
A_{d g} \\
B_{F g} C_{d g} \\
0
\end{array}\right],
\end{aligned}
$$

$$
\begin{aligned}
\widetilde{B}_{g} & =\left[\begin{array}{ccc}
B_{g} & E_{g} & F_{g} \\
0 & B_{F g} D_{g} & B_{F g} H_{g} \\
0 & 0 & B_{w f g}
\end{array}\right], \\
\widetilde{C}_{g} & =\left[\begin{array}{lll}
D_{F g} C_{g} & C_{F g} & -C_{w f g}
\end{array}\right], \\
\widetilde{C}_{d g} & =D_{F g} C_{d g}, \\
\widetilde{D}_{g} & =\left[\begin{array}{lll}
0 & D_{F g} D_{g} & D_{F g} H_{g}-D_{w f g}
\end{array}\right], \\
E & =\left[\begin{array}{lll}
I & 0 & 0
\end{array}\right] .
\end{aligned}
$$

In order to study the next step of progress, we first recommend the definitions of stochastic stability of the Markovian jump system for system (9).

Definition 3 (see [2]). The filtering error system (9) is said to be randomly stable if for $\psi(k)=0$ and any initial condition $\tilde{x}(0) \in \mathbb{R}^{n_{x}}$ and $r(0) \in S$. Then, the following holds: $E\left\{\sum_{k=0}^{\infty}\|\widetilde{x}(k)\|^{2} \mid \widetilde{x}(0), r(0)\right\}<\infty$.

Definition 4 (see [6]). Given the signal $\psi(k) \in l_{2}[0, \infty)$ and a scalar $\gamma>0$, system (9) is randomly stable with an $\mathscr{H}_{\infty}$ disturbance attenuation performance index $\gamma$ if the following two conditions are satisfied.

(1) When $\psi(k)=0, k \geq 0$, system (9) is randomly stable in the sense of Definition 3.

(2) When $\psi(k) \neq 0, k \geq 0$, under zero initial conditions, the following inequality holds:

$$
E\left\{\sum_{k=0}^{\infty}\|e(k)\|^{2}\right\}<\gamma^{2} E\left\{\sum_{k=0}^{\infty}\|\psi(k)\|^{2}\right\} .
$$

To sum up, the main process of the fault detection filter design is to determine the filter matrices $\left\{A_{F g}, B_{F g}, C_{F g}, D_{F g}\right\}$ in system (7), such that the filtering error system (9) is stochastically stable with a reliable $\mathscr{H}_{\infty}$ performance level index. Moreover, in order to detect the fault $f(k)$, the residual evaluation function is designed as $J(\widehat{r}(k))=$ $\sqrt{\sum_{k=k_{0}}^{k_{0}+L} \widehat{r}^{T}(k) \widehat{r}(k)}$, where $k_{0}$ refers to the initial evaluation time instant. The fault $f(k)$ can be detected by the following steps.

(i) The threshold is selected as $J_{\text {th }} \triangleq \sup _{d \in l_{2}, f=0} E[J(\widehat{r}(k))]$.

(ii) Based on the above design, the occurrence of fault $f(k)$ can be judged by the following logical relationship.

When $J(\widehat{r}(k)) \geq J_{\text {th }}$, there are some faults, we should give an alarm; when $J(\widehat{r}(k))<J_{\text {th }}$, there are no faults.

Lemma 5 (see $[27,28])$. For given positive integers $m, n$, a scalar $\beta \in(0,1)$, and two matrices $V_{1} \in \mathbb{S}_{n}^{+}$and $V_{2} \in \mathbb{S}_{m}^{+}$. Introduce the matrix $\Theta(\beta)$ as

$$
\Theta(\beta)=\left[\begin{array}{cc}
\frac{1}{\beta} V_{1} & 0 \\
0 & \frac{1}{1-\beta} V_{2}
\end{array}\right],
$$


If there exists a matrix $X \in \mathbf{R}^{n \times m}$ such that

$$
\Theta_{m}=\left[\begin{array}{cc}
V_{1} & X \\
* & V_{2}
\end{array}\right] \geq 0,
$$

then the following inequality holds:

$$
\Theta(\beta) \geq \Theta_{m} .
$$

Lemma 6 (Wirtinger-based inequality $[29,30])$. For a given symmetric positive definite matrix $V \in \mathbf{S}_{n}^{+}$, any sequence of discrete-time variable $x$ in $[-h, 0] \cap \mathbb{Z} \rightarrow \mathbb{R}^{n}$, where $h \geq 1$, the following inequality holds:

$$
\sum_{g=-h+1}^{0} Z^{T}(g) V Z(g) \geq \frac{1}{h}\left[\begin{array}{c}
Z_{0} \\
Z_{1}
\end{array}\right]^{T}\left[\begin{array}{cc}
V & 0 \\
0 & 3 V
\end{array}\right]\left[\begin{array}{c}
Z_{0} \\
Z_{1}
\end{array}\right],
$$

where $Z(g)=x(g)-x(g-1), Z_{0}=x(0)-x(-h), Z_{1}=$ $x(0)+x(-h)-(2 /(h+1)) \sum_{g=-h}^{0} x(g)$. The set $\mathbf{S}_{n}^{+}$refers to the set of symmetric positive definite matrices.

\section{Main Results}

In the this section, we will first introduce the stability criterion for the filtering error system (9) with polytopic uncertain TRs and time-varying delays; then we will further research on the design of the reduced-order FD filter for polytopic uncertain MJLS with time-varying delays.

\subsection{FD Filtering Stochastic Stability Condition for Discrete}

Time MJLSs with Polytopic Uncertain TRs and TimeVarying Delays

Theorem 7. Given scalars $1 \leq d_{1} \leq d_{2}$, then, for any delay $d(k)$, the discrete-time error augmented system (9) with deficient transition probabilities is randomly stable if there exist positive-definite symmetric matrices $P_{g}, Q_{1}, Q_{2}, Q_{3}, Z_{1}, Z_{2} \in$ $\mathbb{R}^{n \times n}$, and a matrix $X \in \mathbb{R}^{2 n \times 2 n}$ such that the following inequalities hold for any $g=1, \ldots, N$ :

$$
\begin{aligned}
& \Pi_{g} \\
& =\left[\begin{array}{cccccccc}
\Lambda_{11} & \Lambda_{12} & 0 & 0 & 2 \bar{Z}_{1} & 0 & 0 & \Lambda_{18} \\
* & \Lambda_{22} & 0 & 0 & 0 & 0 & 2 \widetilde{\bar{Z}}_{2} & \Lambda_{28} \\
* & * & \Lambda_{33} & 0 & 2 \bar{Z}_{1} & 0 & 0 & 0 \\
* & * & * & \Lambda_{44} & 0 & 0 & 2 \overline{\bar{Z}}_{2} & 0 \\
* & * & * & * & -4 \bar{Z}_{1} & 0 & 0 & 0 \\
* & * & * & * & * & -4 \widetilde{\bar{Z}}_{2} & 0 & 0 \\
* & * & * & * & * & * & -4 \widetilde{\bar{Z}}_{2} & 0 \\
* & * & * & * & * & * & * & \Lambda_{88}
\end{array}\right] \\
& <0, \\
& \widetilde{\bar{Z}}_{2}=\left[\begin{array}{cc}
\bar{Z}_{2} & X \\
* & \bar{Z}_{2}
\end{array}\right] \geq 0,
\end{aligned}
$$

where

$$
\begin{aligned}
\bar{Z}_{1}= & {\left[\begin{array}{cc}
Z_{1} & 0 \\
0 & 3 Z_{1}
\end{array}\right], } \\
\bar{Z}_{2}= & {\left[\begin{array}{cc}
Z_{2} & 0 \\
0 & 3 Z_{2}
\end{array}\right], } \\
\Lambda_{11}= & \widetilde{A}_{g}^{T} \bar{P}_{j} \widetilde{A}_{g}-P_{g} \\
& +E^{T}\left(\left(1+d_{12}\right) Q_{1}+Q_{2}+Q_{3}\right) E \\
& +A_{g}^{T}\left(d_{1}^{2} Z_{1}+d_{12}^{2} Z_{2}\right) A_{g}+\widetilde{C}_{g}^{T} \widetilde{C}_{g}, \\
\Lambda_{12}= & \widetilde{A}_{g}^{T} \bar{P}_{j} \widetilde{A}_{g}+A_{g}^{T}\left(d_{1}^{2} Z_{1}+d_{12}^{2} Z_{2}\right) A_{d g}+\widetilde{C}_{g}^{T} \widetilde{C}_{d g}, \\
\Lambda_{22}= & \widetilde{A}_{d g}^{T} \bar{P}_{j} \widetilde{A}_{d g}+A_{d g}^{T}\left(d_{1}^{2} Z_{1}+d_{12}^{2} Z_{2}\right) A_{d g}-Q_{1} \\
& -4 \widetilde{\bar{Z}}_{2}+\widetilde{C}_{d g}^{T} \widetilde{C}_{d g}, \\
\Lambda_{33}= & -Q_{2}-2 \bar{Z}_{1}-2 \widetilde{\bar{Z}}_{2}, \\
\Lambda_{44}= & -Q_{3}-2 \widetilde{\bar{Z}}_{2}, \\
\Lambda_{18}= & \widetilde{C}_{g}^{T} \widetilde{D}_{g}, \\
\Lambda_{28}= & \widetilde{C}_{d g}^{T} \widetilde{D}_{g}, \\
\Lambda_{88}= & \widetilde{D}_{g}^{T} \widetilde{D}_{g}-\gamma^{2} I, \\
\bar{P}_{j}:= & \sum_{j \in I_{k}^{g}} \lambda_{g j} P_{j}+\sum_{j \in I_{u c}^{g}} \widetilde{\lambda}_{g j}^{l} P_{j}+\widehat{\lambda}_{u k}^{g l} P_{j}, \\
\widehat{\lambda}_{u k}^{g l}:= & 1-\sum_{j \in I_{k}^{g}} \lambda_{g j}-\sum_{j \in I_{u c}^{g}} \widetilde{\lambda}_{g j}^{l} .
\end{aligned}
$$

Proof. When $\psi(k)=0$, we consider the following LyapunovKrasovskii functional:

$$
V(g, \tilde{x}(k)):=\sum_{i=1}^{5} V_{i}(g, \tilde{x}(k)),
$$

with

$$
\begin{aligned}
V_{1}(g, \tilde{x}(k)) & :=\widetilde{x}^{T}(k) P_{g} \tilde{x}(k), \\
V_{2}(g, \widetilde{x}(k)) & :=\sum_{j=k-d(k)}^{k-1} \tilde{x}^{T}(j) E^{T} Q_{1} E \tilde{x}(j), \\
V_{3}(g, \widetilde{x}(k)) & :=\sum_{j=k-d_{1}}^{k-1} \tilde{x}^{T}(j) E^{T} Q_{2} E \tilde{x}(j) \\
& +\sum_{j=k-d_{2}}^{k-1} \tilde{x}^{T}(j) E^{T} Q_{3} E \tilde{x}(j),
\end{aligned}
$$




$$
\begin{aligned}
& V_{4}(g, \tilde{x}(k)):=\sum_{m=-d_{2}+1}^{-d_{1}} \sum_{j=k-d(k)}^{k-1} \tilde{x}^{T}(j) E^{T} Q_{1} E \tilde{x}(j), \\
& V_{5}(g, \tilde{x}(k)):=d_{1} \sum_{m=-d_{1}}^{-1} \sum_{j=k+m}^{k-1}(\tilde{x}(j+1)-\tilde{x}(j))^{T} \\
& \cdot E^{T} Z_{1} E(\tilde{x}(j+1)-\tilde{x}(j)) \\
& +d_{12} \sum_{m=-d_{2}}^{-d_{1}-1} \sum_{j=k+m}^{k-1}(\tilde{x}(j+1)-\tilde{x}(j))^{T} \\
& \cdot E^{T} Z_{2} E(\tilde{x}(j+1)-\tilde{x}(j)),
\end{aligned}
$$

where $P_{g}=P_{g}^{T}>0, Q_{i}=Q_{i}^{T}>0, i \in\{1,2,3\}, Z_{j}=Z_{j}^{T}>0$, $j \in\{1,2\}$ are parameter-dependent Lyapunov matrices to be determined. obtain

Calculating the difference of $V(g, \tilde{x}(k))$ for each $g$, we

$$
\begin{aligned}
& \Delta V_{1}=\tilde{x}^{T}(k+1) \sum_{j=1}^{N} \lambda_{g j} P_{j} \tilde{x}(k+1)-\tilde{x}^{T}(k) P_{g} \tilde{x}(k) \\
& =\left(\widetilde{A}_{g} \tilde{x}(k)+\widetilde{A}_{d g} E \tilde{x}(k-d(k))\right)^{T}\left(\sum_{j=1}^{N} \lambda_{g j} P_{j}\right) \\
& \cdot\left(\widetilde{A}_{g} \tilde{x}(k)+\widetilde{A}_{d g} E \tilde{x}(k-d(k))\right)-\tilde{x}^{T}(k) P_{g} \tilde{x}(k), \\
& \Delta V_{2} \leq \tilde{x}^{T}(k) E^{T} Q_{1} E \tilde{x}(k)-\tilde{x}^{T}(k-d(k)) \\
& \cdot E^{T} Q_{1} E \tilde{x}(k-d(k))+\sum_{j=k-d_{2}+1}^{k-d_{1}} \tilde{x}^{T}(j) E^{T} Q_{1} E \tilde{x}(j), \\
& \Delta V_{3}=\tilde{x}^{T}(k) E^{T}\left(Q_{2}+Q_{3}\right) E \tilde{x}(k)-\tilde{x}^{T}\left(k-d_{1}\right) \\
& \cdot E^{T} Q_{2} E \tilde{x}\left(k-d_{1}\right)-\tilde{x}^{T}\left(k-d_{2}\right) \\
& \text { - } E^{T} Q_{3} E \tilde{x}\left(k-d_{2}\right) \text {, } \\
& \Delta V_{4}=d_{12} \tilde{x}^{T}(k) E^{T} Q_{1} E \tilde{x}(k)-\sum_{j=k-d_{2}+1}^{k-d_{1}} \tilde{x}^{T}(j) \\
& \cdot E^{T} Q_{1} E \tilde{x}(j) \\
& \Delta V_{5}=(E(\tilde{x}(k+1)-\tilde{x}(k)))^{T}\left(d_{1}^{2} Z_{1}+d_{12}^{2} Z_{2}\right) \\
& \cdot(E(\tilde{x}(k+1)-\tilde{x}(k))) \\
& -d_{1} \sum_{j=k-d_{1}}^{k-1}(E(\tilde{x}(j+1)-\tilde{x}(j)))^{T} \\
& \cdot Z_{1}(E(\tilde{x}(j+1)-\tilde{x}(j)))
\end{aligned}
$$

$$
\begin{aligned}
& -d_{12} \sum_{j=k-d_{2}}^{k-d_{1}-1}(E(\tilde{x}(j+1)-\tilde{x}(j)))^{T} \\
& \cdot Z_{2}(E(\tilde{x}(j+1)-\tilde{x}(j))) .
\end{aligned}
$$

On the other hand, according to Lemmas 5 and 6 , the following inequality is given:

$$
\begin{aligned}
& -d_{1} \sum_{j=k-d_{1}}^{k-1}(E(\tilde{x}(j+1)-\tilde{x}(j)))^{T} \\
& \cdot Z_{1}(E(\tilde{x}(j+1)-\tilde{x}(j))) \leq-\theta_{1}^{T} \bar{Z}_{1} \theta_{1} \\
& =\eta_{1}^{T} \Lambda_{1}^{T} \bar{Z}_{1} \Lambda_{1} \eta_{1},
\end{aligned}
$$

where

$$
\begin{aligned}
& \theta_{1}=\left[\begin{array}{c}
x(k)-x\left(k-d_{1}\right) \\
x(k)+x\left(k-d_{1}\right)-\frac{2}{d_{1}+1} \sum_{j=k-d_{1}}^{k} x(j)
\end{array}\right], \\
& \bar{Z}_{1}=\left[\begin{array}{cc}
Z_{1} & 0 \\
0 & 3 Z_{1}
\end{array}\right],
\end{aligned}
$$$$
\eta_{1}(k)=\left[\begin{array}{c}
\tilde{x}(k) \\
x(k-d(k)) \\
x\left(k-d_{1}\right) \\
x\left(k-d_{2}\right) \\
\frac{1}{d_{1}+1} \sum_{j=k-d_{1}}^{k} x(j) \\
\frac{1}{d(k)-d_{1}+1} \sum_{j=k-d(k)}^{k-d_{1}} x(j) \\
\frac{1}{d_{2}-d(k)+1} \sum_{j=k-d_{2}}^{k} x(j)
\end{array}\right],
$$

$$
\Lambda_{1}=\left[\begin{array}{ccccccc}
I & 0 & -I & 0 & 0 & 0 & 0 \\
I & 0 & I & 0 & -2 I & 0 & 0
\end{array}\right] \text {, }
$$$$
-d_{12} \sum_{j=k-d_{2}}^{k-d_{1}-1}(E(\tilde{x}(j+1)-\tilde{x}(j)))^{T}
$$

$$
\cdot Z_{2}(E(\tilde{x}(j+1)-\tilde{x}(j)))
$$

$$
=-d_{12} \sum_{j=k-d(k)}^{k-d_{1}-1}(E(\tilde{x}(j+1)-\tilde{x}(j)))^{T}
$$$$
\cdot Z_{2}(E(\tilde{x}(j+1)-\tilde{x}(j)))
$$$$
-d_{12} \sum_{j=k-d_{2}}^{k-d(k)-1}(E(\tilde{x}(j+1)-\tilde{x}(j)))^{T}
$$ 


$$
\begin{aligned}
& \cdot Z_{2}(E(\tilde{x}(j+1)-\tilde{x}(j))) \leq-\frac{d_{12}}{d(k)-d_{1}} \theta_{2}^{T} \bar{Z}_{2} \theta_{2} \\
& -\frac{d_{12}}{d_{2}-d(k)} \theta_{3}^{T} \bar{Z}_{2} \theta_{3} \\
& =-\eta_{1}^{T} \Lambda_{2}^{T}\left[\begin{array}{cc}
\frac{d_{12}}{d(k)-d_{1}} \bar{Z}_{2} & 0 \\
0 & \frac{d_{12}}{d_{2}-d(k)} \bar{Z}_{2}
\end{array}\right] \Lambda_{2} \eta_{1} \\
& \leq-\eta_{1}^{T} \Lambda_{2}^{T}\left[\begin{array}{cc}
\bar{Z}_{2} & X \\
* & \bar{Z}_{2}
\end{array}\right] \Lambda_{2} \eta_{1},
\end{aligned}
$$$$
\Pi_{1 g}=\left[\begin{array}{ccccccc}
\bar{\Lambda}_{11} & \bar{\Lambda}_{12} & 0 & 0 & 2 \bar{Z}_{1} & 0 & 0 \\
* & \bar{\Lambda}_{22} & 0 & 0 & 0 & 0 & 2 \widetilde{\bar{Z}}_{2} \\
* & * & \Lambda_{33} & 0 & 2 \bar{Z}_{1} & 0 & 0 \\
* & * & * & \Lambda_{44} & 0 & 0 & 2 \widetilde{\bar{Z}}_{2} \\
* & * & * & * & -4 \bar{Z}_{1} & 0 & 0 \\
* & * & * & * & * & -4 \widetilde{\bar{Z}}_{2} & 0 \\
* & * & * & * & * & * & -4 \widetilde{\bar{Z}}_{2}
\end{array}\right],
$$

where

$$
\begin{aligned}
& \theta_{2} \\
& =\left[\begin{array}{c}
x\left(k-d_{1}\right)-x(k-d(k)) \\
x\left(k-d_{1}\right)+x(k-d(k))-\frac{2}{d(k)-d_{1}+1} \sum_{j=k-d(k)}^{k-d_{1}} x(j)
\end{array}\right], \\
& \theta_{3} \\
& =\left[\begin{array}{c}
x(k-d(k))-x\left(k-d_{2}\right) \\
x(k-d(k))+x\left(k-d_{2}\right)-\frac{2}{d_{2}-d(k)+1} \sum_{j=k-d_{2}}^{k-d(k)} x(j)
\end{array}\right], \\
& \bar{Z}_{2}=\left[\begin{array}{cc}
Z_{2} & 0 \\
0 & 3 Z_{2}
\end{array}\right] \text {, } \\
& \Lambda_{2}=\left[\begin{array}{ccccccc}
0 & -I & I & 0 & 0 & 0 & 0 \\
0 & I & I & 0 & 0 & -2 I & 0 \\
0 & I & 0 & -I & 0 & 0 & 0 \\
0 & I & 0 & I & 0 & 0 & -2 I
\end{array}\right] \text {. }
\end{aligned}
$$

Using this and combining (20)-(24), we have

$$
\begin{aligned}
\Delta V & =\Delta V_{1}+\Delta V_{2}+\Delta V_{3}+\Delta V_{4}+\Delta V_{5} \\
& \leq\left(\widetilde{A}_{g} \tilde{x}(k)+\widetilde{A}_{d g} E \tilde{x}(k-d(k))\right)^{T}\left(\sum_{j=1}^{N} \lambda_{g j} P_{j}\right) \\
& \cdot\left(\widetilde{A}_{g} \tilde{x}(k)+\widetilde{A}_{d g} E \tilde{x}(k-d(k))\right)-\tilde{x}^{T}(k) P_{g} \tilde{x}(k) \\
& +\tilde{x}^{T}(k) E^{T} Q_{1} E \tilde{x}(k)-\tilde{x}^{T}(k-d(k)) \\
& \cdot E^{T} Q_{1} E \tilde{x}(k-d(k))+\tilde{x}^{T}(k) E^{T}\left(Q_{2}+Q_{3}\right) E \tilde{x}(k) \\
& -\tilde{x}^{T}\left(k-d_{1}\right) E^{T} Q_{2} E \tilde{x}\left(k-d_{1}\right)-\tilde{x}^{T}\left(k-d_{2}\right) \\
& \cdot E^{T} Q_{3} E \tilde{x}\left(k-d_{2}\right)+d_{12} \tilde{x}^{T}(k) E^{T} Q_{1} E \tilde{x}(k) \\
& +(E(\tilde{x}(k+1)-\tilde{x}(k)))^{T}\left(d_{1}^{2} Z_{1}+d_{12}^{2} Z_{2}\right) \\
& \cdot(E(\tilde{x}(k+1)-\tilde{x}(k)))-\eta_{1}^{T}(k) \Lambda_{1}^{T} \bar{Z}_{1} \Lambda_{1} \eta_{1}(k) \\
& -\eta_{1}^{T}(k) \Lambda_{2}^{T} \widetilde{\bar{Z}}_{2} \Lambda_{2} \eta_{1}(k)=\eta_{1}^{T}(k) \Pi_{1 g} \eta_{1}(k),
\end{aligned}
$$

where $\Lambda_{33}, \Lambda_{44}$ are defined in (19) and

$$
\begin{aligned}
\bar{\Lambda}_{11}= & \widetilde{A}_{g}^{T}\left(\sum_{j=1}^{N} \lambda_{g j} P_{j}\right) \widetilde{A}_{g}-P_{g} \\
& +E^{T}\left(\left(1+d_{12}\right) Q_{1}+Q_{2}+Q_{3}\right) E \\
& +A_{g}^{T}\left(d_{1}^{2} Z_{1}+d_{12}^{2} Z_{2}\right) A_{g}, \\
\bar{\Lambda}_{12}= & \widetilde{A}_{g}^{T}\left(\sum_{j=1}^{N} \lambda_{g j} P_{j}\right) \widetilde{A}_{g}+A_{g}^{T}\left(d_{1}^{2} Z_{1}+d_{12}^{2} Z_{2}\right) A_{d g}, \\
\bar{\Lambda}_{22}= & \widetilde{A}_{d g}^{T}\left(\sum_{j=1}^{N} \lambda_{g j} P_{j}\right) \widetilde{A}_{d g} \\
& +A_{d g}^{T}\left(d_{1}^{2} Z_{1}+d_{12}^{2} Z_{2}\right) A_{d g}-Q_{1}-4 \widetilde{\bar{Z}}_{2} .
\end{aligned}
$$

Because $\Pi_{1 g}<0$, we can see that $\Delta V \leq 0$ is holding. We can conclude that $\tilde{x}(0) \in \mathbb{R}^{n_{x}}$ and $r(0) \in S$. Then, the following holds:

$$
E\left\{\sum_{k=0}^{\infty}\|\tilde{x}(k)\|^{2} \mid \tilde{x}(0), r(0)\right\}<\infty .
$$

The stochastic stability is satisfied.

When $\psi(k) \neq 0$, we set

$$
\eta_{2}(k)=\left[\begin{array}{c}
\tilde{x}(k) \\
x(k-d(k)) \\
x\left(k-d_{1}\right) \\
x\left(k-d_{2}\right) \\
\frac{1}{d_{1}+1} \sum_{j=k-d_{1}}^{k} x(j) \\
\frac{1}{d(k)-d_{1}+1} \sum_{j=k-d(k)}^{k-d_{1}} x(j) \\
\frac{1}{d_{2}-d(k)+1} \sum_{j=k-d_{2}}^{k(k)} x(j)
\end{array}\right],
$$




$$
\begin{aligned}
J= & E\left\{\sum _ { k = 0 } ^ { \infty } \left[e^{T}(k) e(k)-\gamma^{2} \psi^{T}(k) \psi(k)\right.\right. \\
& +\Delta V(\tilde{x}(k), g)]\}-\left.E(V(\tilde{x}(k), g))\right|_{k \rightarrow \infty} \\
& \leq \sum_{k=0}^{\infty}\left[e^{T}(k) e(k)-\gamma^{2} \psi^{T}(k) \psi(k)\right. \\
& +\Delta V(\tilde{x}(k), g)] \leq \sum_{k=0}^{\infty} \eta_{2}^{T}(k) \Pi_{2 g} \eta_{2}(k),
\end{aligned}
$$

where

$$
\begin{aligned}
& \Pi_{2 g} \\
& =\left[\begin{array}{cccccccc}
\Lambda_{11}^{\prime} & \Lambda_{12}^{\prime} & 0 & 0 & 2 \bar{Z}_{1} & 0 & 0 & \Lambda_{18} \\
* & \Lambda_{22}^{\prime} & 0 & 0 & 0 & 0 & 2 \overline{\bar{Z}}_{2} & \Lambda_{28} \\
* & * & \Lambda_{33} & 0 & 2 \bar{Z}_{1} & 0 & 0 & 0 \\
* & * & * & \Lambda_{44} & 0 & 0 & 2 \overline{\bar{Z}}_{2} & 0 \\
* & * & * & * & -4 \bar{Z}_{1} & 0 & 0 & 0 \\
* & * & * & * & * & -4 \overline{\bar{Z}}_{2} & 0 & 0 \\
* & * & * & * & * & * & -4 \overline{\bar{Z}}_{2} & 0 \\
* & * & * & * & * & * & * & \Lambda_{88}
\end{array}\right],
\end{aligned}
$$

where $\Lambda_{18}, \Lambda_{28}, \Lambda_{88}$ are defined in (19) and

$$
\begin{aligned}
\Lambda_{11}^{\prime}= & \widetilde{A}_{g}^{T}\left(\sum_{j=1}^{N} \lambda_{g j} P_{j}\right) \widetilde{A}_{g}-P_{g} \\
& +E^{T}\left(\left(1+d_{12}\right) Q_{1}+Q_{2}+Q_{3}\right) E \\
& +A_{g}^{T}\left(d_{1}^{2} Z_{1}+d_{12}^{2} Z_{2}\right) A_{g}+\widetilde{C}_{g}^{T} \widetilde{C}_{g} \\
\Lambda_{12}^{\prime}= & \widetilde{A}_{g}^{T}\left(\sum_{j=1}^{N} \lambda_{g j} P_{j}\right) \widetilde{A}_{g}+A_{g}^{T}\left(d_{1}^{2} Z_{1}+d_{12}^{2} Z_{2}\right) A_{d g} \\
& +\widetilde{C}_{g}^{T} \widetilde{C}_{d g}, \\
\Lambda_{22}^{\prime}= & \widetilde{A}_{d g}^{T}\left(\sum_{j=1}^{N} \lambda_{g j} P_{j}\right) \widetilde{A}_{d g} \\
& +A_{d g}^{T}\left(d_{1}^{2} Z_{1}+d_{12}^{2} Z_{2}\right) A_{d g}-Q_{1}-4 \widetilde{\bar{Z}}_{2} \\
& +\widetilde{C}_{d g}^{T} \widetilde{C}_{d g} .
\end{aligned}
$$

When the polytopic uncertain transition rates are simultaneously considered, we can conclude that formulas (16)-(17) hold for any nonzero condition. This proof of Theorem 7 is completed.

\subsection{Design of Reduced-Order FD Filter for Polytopic Discrete-Time MJLSs with Time-Varying Delays}

Theorem 8. Consider system (1) with polytopic uncertain transition information and time-varying delays, for given $\gamma>$ 0 , if there exist matrices $X, M_{g j}:=\left[\begin{array}{cc}M_{g j(1)} & W M_{g j(2)} \\ M_{g j(3)} & M_{g j(2)}\end{array}\right], \forall g, j \in$ $I, W:=\left[\begin{array}{ll}I_{n_{r}} & 0_{n_{r} \times\left(n_{x}-n_{r}\right)}\end{array}\right]^{T}, \overline{\bar{Z}}_{2} \geq 0$ and positive-definite symmetric matrices $P_{g}, Q_{1}, Q_{2}, Q_{3}, Z_{1}, Z_{2}$ satisfy the following LMIs,

$$
\widetilde{\Pi}_{g}=\left[\begin{array}{ccccc}
\Phi_{1} & \Phi_{2} & \Phi_{3} & \Phi_{4} & \Phi_{5} \\
* & -Z_{1} & 0 & 0 & 0 \\
* & * & -Z_{2} & 0 & 0 \\
* & * & * & -I & 0 \\
* & * & * & * & \Phi_{6}
\end{array}\right]<0
$$

where

$$
\begin{aligned}
& \Phi_{1} \\
& =\left[\begin{array}{cccccccc}
\widetilde{\Lambda}_{11} & 0 & 0 & 0 & 2 \bar{Z}_{1} & 0 & 0 & 0 \\
* & \widetilde{\Lambda}_{22} & 0 & 0 & 0 & 0 & 2 \widetilde{\bar{Z}}_{2} & 0 \\
* & * & \Lambda_{33} & 0 & 2 \bar{Z}_{1} & 0 & 0 & 0 \\
* & * & * & \Lambda_{44} & 0 & 0 & 2 \widetilde{\bar{Z}}_{2} & 0 \\
* & * & * & * & -4 \bar{Z}_{1} & 0 & 0 & 0 \\
* & * & * & * & * & -4 \widetilde{\bar{Z}}_{2} & 0 & 0 \\
* & * & * & * & * & * & -4 \widetilde{\bar{Z}}_{2} & 0 \\
* & * & * & * & * & * & * & -\gamma^{2} I
\end{array}\right], \\
& \Phi_{2}=\left[\begin{array}{llllllll}
d_{1} Z_{1} A_{g} & d_{1} Z_{1} A_{d g} & 0 & 0 & 0 & 0 & 0 & 0
\end{array}\right]^{T} \text {, } \\
& \Phi_{3}=\left[\begin{array}{llllllll}
d_{12} Z_{2} A_{g} & d_{12} Z_{2} A_{d g} & 0 & 0 & 0 & 0 & 0 & 0
\end{array}\right]^{T}, \\
& \Phi_{4}=\left[\begin{array}{llllllll}
\widetilde{C}_{g} & \widetilde{C}_{d g} & 0 & 0 & 0 & 0 & 0 & \widetilde{D}_{g}
\end{array}\right]^{T}, \\
& \Phi_{5}=\left[\begin{array}{llllllll}
\Lambda_{112}^{T} & \Lambda_{212}^{T} & 0 & 0 & 0 & 0 & 0 & 0
\end{array}\right]^{T} \text {, } \\
& \widetilde{\Lambda}_{11}=E^{T}\left(\left(1+d_{12}\right) Q_{1}+Q_{2}+Q_{3}\right) E-\bar{Z}_{1}-P_{g}, \\
& \widetilde{\Lambda}_{22}=-Q_{1}-4 \widetilde{\bar{Z}}_{2} \\
& \Lambda_{112}=\left[\sqrt{\lambda_{g S_{k(1)}^{(g)}}} A_{g S_{k(1)}^{(g)}}^{T}, \ldots, \sqrt{\lambda_{g S_{k(s)}^{(g)}}} A_{g S_{k(s)}^{(g)}}^{T},\right. \\
& \left.\sqrt{\widetilde{\lambda}_{g S_{u c(1)}^{(g)}}^{(g)}} A_{g S_{u c(1)}^{(g)}}^{T}, \ldots, \sqrt{\tilde{\bar{\lambda}}_{g S_{u c(v)}^{(g)}}^{(g)}} A_{g S_{u c(v)}^{(g)}}^{T}, \sqrt{\widehat{\lambda}_{u k}^{(g l)}} A_{g S_{u k}^{(g)}}^{T}\right], \\
& \Lambda_{212}=\left[\sqrt{\lambda_{g S_{k(1)}^{(g)}}} A_{d g S_{k(1)}^{(g)}}^{T}, \ldots, \sqrt{\lambda_{g S_{k(s)}^{(g)}}} A_{d g S_{k(s)}^{T}}^{T},\right. \\
& \left.\sqrt{\tilde{\bar{\lambda}}_{g S_{u c(1)}^{(g)}}^{(g)}} A_{d g S_{u c(1)}^{(g)}}^{T}, \ldots, \sqrt{\tilde{\bar{\lambda}}_{g S_{u c(v)}^{(g)}}^{(g)}} A_{d g S_{u c(v)}^{(g)}}^{T}, \sqrt{\hat{\lambda}_{u k}^{(g l)}} A_{d g S_{u k}^{(g)}}^{T}\right] \text {, }
\end{aligned}
$$




$$
\begin{aligned}
& \Phi_{6}=\operatorname{diag}\left\{H_{g S_{k(1)}^{(g)}}, \ldots, H_{g S_{k(s)}^{(g)}}, \ldots, H_{g S_{u c(1)}^{(g)}}, \ldots, H_{g S_{u c(v)}^{(g)}},\right. \\
& \left.H_{g S_{u k}^{(g)}}\right\} \\
& H_{g j}=P_{j}-M_{g j}-M_{g j}^{T},
\end{aligned}
$$

then the FD filter (7) matrices $\left(A_{F g}, B_{F g}, C_{F g}, D_{F g}\right)$ are found so that the filtering error system (9) is stochastically stable with an $\mathscr{H}_{\infty}$ performance index $\gamma$. Moreover, the designed reducedorder FD filter's gain matrices can be obtained as follows:

$$
\begin{aligned}
& \widetilde{A}_{F g}:=M_{g j(2)} A_{F g}, \\
& \widetilde{B}_{F g}:=M_{g j(2)} B_{F g}, \\
& \widetilde{C}_{F g}:=C_{F g}, \\
& \widetilde{D}_{F g}:=D_{F g} .
\end{aligned}
$$

Proof. According to Theorem 7, the filtering error systems are randomly stable with a guaranteed $\mathscr{H}_{\infty}$ performance $\gamma$ if there exist positive-definite symmetric matrices $P_{g}, g \in$ $I, Q_{1}, Q_{2}, Q_{3}, Z_{1}, Z_{2}$, and a matrix $X$ satisfying the inequalities in (16)-(17). We firstly apply Schur complements to (16) and obtain

$$
\widetilde{\Pi}_{1 g}=\left[\begin{array}{ccccc}
\Phi_{1} & \Phi_{2} & \Phi_{3} & \Phi_{4} & \widetilde{\Phi}_{5} \\
* & -Z_{1} & 0 & 0 & 0 \\
* & * & -Z_{2} & 0 & 0 \\
* & * & * & -I & 0 \\
* & * & * & * & -\widetilde{\Phi}_{6}
\end{array}\right]<0,
$$

where $\Phi_{1}, \Phi_{2}, \Phi_{3}$, and $\Phi_{4}$ are defined in (33) and

$$
\begin{aligned}
& \widetilde{\Phi}_{5}=\left[\begin{array}{llllllll}
\widetilde{\Lambda}_{112}^{T} & \widetilde{\Lambda}_{212}^{T} & 0 & 0 & 0 & 0 & 0 & 0
\end{array}\right]^{T}, \\
& \widetilde{\Phi}_{6}=\operatorname{diag}\left\{P_{S_{k(1)}^{(g)}}^{-1}, \ldots, P_{S_{k(s)}^{(g)}}^{-1}, P_{S_{u c(1)}^{(g)}}^{-1}, \ldots, P_{S_{u c(v)}^{(g)}}^{-1}, P_{S_{u k}^{(g)}}^{-1}\right\}, \\
& \widetilde{\Lambda}_{112}=\widetilde{A}_{g}^{T}\left[\sqrt{\lambda_{g S_{k(1)}^{(g)}}} I, \ldots, \sqrt{\lambda_{g S_{k(s)}^{(g)}}} I, \ldots, \sqrt{\widetilde{\lambda}_{g S_{u c(1)}^{(g)}}^{(g)}} I, \ldots,\right. \\
& \left.\sqrt{\tilde{\lambda}_{g S_{u c(v)}^{(g)}}^{(g)}} I, \sqrt{\widehat{\lambda}_{u k}^{(g l)}} I\right] \\
& \widetilde{\Lambda}_{212}=\widetilde{A}_{d g}^{T}\left[\sqrt{\lambda_{g S_{k(1)}^{(g)}}} I, \ldots, \sqrt{\lambda_{g S_{k(s)}^{(g)}}} I, \ldots, \sqrt{\widetilde{\lambda}_{g S_{u c(1)}^{(g)}}^{(g)}} I, \ldots,\right. \\
& \left.\sqrt{\underset{\tilde{\lambda}_{u c(v)}^{(g)}}{(g)}} I, \sqrt{\widehat{\lambda}_{u k}^{(g l)}} I\right]
\end{aligned}
$$

In order to eliminate the coupling between the system matrices and the Lyapunov matrices in (35), we perform a congruent transformation to (35) by $\operatorname{diag}\left\{I_{(4 n+m)}, I_{n}, I_{n}, I_{q}, \Omega\right\}$, with

$$
\begin{aligned}
& \Omega:=\operatorname{diag}\left\{M_{g S_{k(1)}^{(g)}}, \ldots, M_{g S_{k(s)}^{(g)}}, M_{g S_{u c(1)}^{(g)}}, \ldots, M_{g S_{u c(v)}^{(g)}},\right. \\
& \left.M_{g S_{u k}}\right\} .
\end{aligned}
$$

For an discretionary matrix $M_{g j}, \forall g, j \in I$, the following inequality with $P_{j}>0$ is established:

$$
\left(P_{j}^{T}-M_{g j}^{T}\right) P_{j}^{-1}\left(P_{j}-M_{g j}\right) \geq 0 .
$$

We expand (38) and, then, get the following bounding inequality:

$$
P_{j}^{T}-M_{g j}^{T}-M_{g j} \geq-M_{g j} P_{j}^{-1} M_{g j}^{T} .
$$

Then, based on (39), the following form can be easily obtained:

$$
\widetilde{\Pi}_{2 g}=\left[\begin{array}{ccccc}
\Phi_{1} & \Phi_{2} & \Phi_{3} & \Phi_{4} & \Omega \widetilde{\Phi}_{5} \\
* & -Z_{1} & 0 & 0 & 0 \\
* & * & -Z_{2} & 0 & 0 \\
* & * & * & -I & 0 \\
* & * & * & * & \Phi_{6}
\end{array}\right]<0
$$

For reduced-order FD filter design purpose, we choose the slack matrix $M_{g j}$ as follows:

$$
M_{g j}:=\left[\begin{array}{cc}
M_{g j(1)} & W M_{g j(2)} \\
M_{g j(3)} & M_{g j(4)}
\end{array}\right], \quad g, j \in I,
$$

where

$$
\begin{aligned}
& W:=\left[\begin{array}{ll}
I_{n_{r}} & O_{n_{r} \times\left(n_{x}-n_{r}\right)}
\end{array}\right]^{T}, \\
& M_{g j(1)} \in R^{n_{x} \times n_{x}}, \\
& M_{g j(3)} \in R^{n_{r} \times n_{x}}, \\
& M_{g j(2)} \in R^{n_{r} \times n_{r}}, \\
& M_{g j(4)} \in R^{n_{r} \times n_{r}} .
\end{aligned}
$$

Then, performing the congruent transformation,

$$
\begin{aligned}
& {\left[M_{g j}+M_{g j}^{T}\right]} \\
& \quad=\left[\begin{array}{cc}
M_{g j(1)}+M_{g j(1)}^{T} & W M_{g j(2)}+M_{g j(3)}^{T} \\
* & M_{g j(4)}+M_{g j(4)}^{T}
\end{array}\right],
\end{aligned}
$$

by $\left[\begin{array}{lc}I & 0 \\ * & M_{g j(2)} M_{g j(4)}^{-1}\end{array}\right]$ yields 


$$
\begin{aligned}
& {\left[\begin{array}{cc}
I & 0 \\
* & M_{g j(2)} M_{g j(4)}^{-1}
\end{array}\right]\left[\begin{array}{cc}
M_{g j(1)}+M_{g j(1)}^{T} & W M_{g j(2)}+M_{g j(3)}^{T} \\
* & M_{g j(4)}+M_{g j(4)}^{T}
\end{array}\right]\left[\begin{array}{cc}
I & 0 \\
* & M_{g j(4)}^{-T} M_{g j(2)}^{T}
\end{array}\right]} \\
& \quad=\left[\begin{array}{cc}
M_{g j(1)}+M_{g j(1)}^{T} & W M_{g j(2)} M_{g j(4)}^{-T} M_{g j(2)}^{T}+M_{g j(3)}^{T} M_{g j(4)}^{-T} M_{g j(2)}^{T} \\
* & M_{g j(2)} M_{g j(4)}^{-T} M_{g j(2)}^{T}+M_{g j(2)} M_{g j(4)}^{-1} M_{g j(2)}^{T}
\end{array}\right]=\left[\begin{array}{cc}
M_{g j(1)}+M_{g j(1)}^{T} & W \widetilde{M}_{g j(2)}+\widetilde{M}_{g j(3)}^{T} \\
* & \widetilde{M}_{g j(2)}+\widetilde{M}_{g j(2)}^{T}
\end{array}\right] .
\end{aligned}
$$

Thus, without loss of feasibility, matrix $M_{g j}$ in (41) can directly specify the following general form:

$$
M_{g j}:=\left[\begin{array}{cc}
M_{g j(1)} & W M_{g j(2)} \\
M_{g j(3)} & M_{g j(2)}
\end{array}\right], \quad \forall g, j \in I .
$$

It is shown that the matrix variables $M_{g j(2)}$ can be absorbed directly by the full-order filter gain matrices $A_{F g}$ and $B_{F g}$; then the designed reduced-order FD filter's gain matrices can be obtained as follows (34).

Then we replace matrices $M_{g j}$ given by (34) into (40); finally we can get (32) exactly. This completes the proof.

Remark 9 (fault detection filter design algorithm).

Step 1. Obtain $\gamma_{\min }$ by solving (32) in Theorem 8.

Step 2. If, with $\gamma$ replaced by $\gamma_{\min }$, (32) is feasible for Theorem 8 , we can obtain the locally optimized parameters $\left(A_{F g}, B_{F g}, C_{F g}, D_{F g}\right)$ for the desired full order fault detection filter and $\left(\widetilde{A}_{F g}, \widetilde{B}_{F g}, \widetilde{C}_{F g}, \widetilde{D}_{F g}\right)$ for the desired reducedorder fault detection filter and exit. Otherwise, go to Step 3.

Step 3. Increase $\gamma_{\min }$ by $\Delta \gamma$ where $\Delta \gamma$ is a sufficiently small positive scalar and then solve (32) with the updated $\gamma_{\min }$. Repeat such a procedure until (32) are feasible and therefore obtain the locally optimized filter parameters and the index $\gamma_{\min }$.

Step 4. Stop.

\section{Simulated SRM System}

We now use the proposed method to simulate a four-phase switched reluctance motor (SRM) system investigated in [31] to demonstrate the effectiveness and practicability of the proposed method. The four-phase CSTR system structure is depicted in Figure 1.

The plant state variable is selected as $x=\left[\begin{array}{ll}\theta_{m} & V_{m}\end{array}\right]^{T}$, where $\theta_{m}, V_{m}$ denote the angular position and velocity of the motors. Sampling time $T=0.1$ seconds, and the disturbance variable is selected as $\omega(k)=\left[\begin{array}{ll}0 & \sin \theta_{m}\end{array}\right]^{T}$. The simplified system model is expressed as follows:

$$
\begin{aligned}
& \dot{\theta}_{m}=V_{m}, \\
& \dot{V}_{m}=-\frac{\kappa_{e}}{J_{m}} \sin \theta_{m}-\frac{a_{i}}{J_{m}} V_{m}+\frac{b_{i}}{J_{m}} u_{i}, \quad i=1,2,3,4,
\end{aligned}
$$

where $J_{m}$ denotes the inertia of the motor. $\kappa_{e}>0$ is the elasticity. $u_{i}$ is the voltage applied to the motor of phase $i . a_{i}$ is the related viscous friction, and $b_{i}$ is the amplifier gain.

The above SRM system can be represented by MJS with four operation modes and the following parameter matrices:

$$
\begin{aligned}
& {\left[\begin{array}{c|c|c|c|c}
A_{1} & A_{d 1} & B_{1} & E_{1} & F_{1} \\
\hline C_{1} & C_{d 1} & 0 & D_{1} & H_{1}
\end{array}\right]} \\
& =\left[\begin{array}{cc|cc|c|c|c}
1 & 0.017 & -0.201 & -0.015 & 0.002 & -0.001 & 0.019 \\
0.012 & 0.434 & 0.036 & -0.069 & 0.437 & -0.005 & 0.967 \\
\hline 1.005 & 2.001 & 0.109 & 0.125 & 0 & 0.031 & 0
\end{array}\right] \text {, } \\
& {\left[\begin{array}{c|c|c|c|c}
A_{2} & A_{d 2} & B_{2} & E_{2} & F_{2} \\
\hline C_{2} & C_{d 2} & 0 & D_{2} & H_{2}
\end{array}\right]} \\
& =\left[\begin{array}{cc|cc|c|c|c}
1 & 0.023 & -0.204 & 0.021 & 0.003 & -0.001 & 0.019 \\
0.016 & 0.411 & -0.063 & -0.071 & 0.327 & -0.005 & 0.967 \\
\hline 1.002 & 2.003 & 0.121 & 0.123 & 0 & 0.029 & 0
\end{array}\right] \text {, } \\
& {\left[\begin{array}{c|c|c|c|c}
A_{3} & A_{d 3} & B_{3} & E_{3} & F_{3} \\
\hline C_{3} & C_{d 3} & 0 & D_{3} & H_{3}
\end{array}\right]} \\
& =\left[\begin{array}{cc|cc|c|c|c}
1 & 0.025 & -0.183 & -0.049 & 0.006 & -0.001 & 0.019 \\
0.023 & 0.512 & 0.051 & -0.062 & 0.601 & -0.005 & 0.967 \\
\hline 1.006 & 2.002 & 0.115 & 0.097 & 0 & -0.113 & 0
\end{array}\right] \text {, } \\
& {\left[\begin{array}{c|c|c|c|c}
A_{4} & A_{d 4} & B_{4} & E_{4} & F_{4} \\
\hline C_{4} & C_{d 4} & 0 & D_{4} & H_{4}
\end{array}\right]} \\
& =\left[\begin{array}{cc|cc|c|c|c}
1 & 0.031 & -0.210 & 0.027 & 0.001 & -0.001 & 0.019 \\
0.018 & 0.394 & -0.062 & -0.073 & 0.193 & -0.005 & 0.967 \\
\hline 1.003 & 2.005 & 0.139 & 0.108 & 0 & 0.112 & 0
\end{array}\right] \text {, }
\end{aligned}
$$

Weighted transfer function matrix is $W_{f}(z)=$ $0.5 z /(0.2 z+1)$, in which state space is realized as follows:

$$
\begin{aligned}
x_{f}(k+1) & =x_{f}(k)+0.25 f(k), \\
\bar{f}(k) & =0.51 x_{f}(k)+f(k) .
\end{aligned}
$$

In order to make the simulation simplification, we consider $1 \leq d(k) \leq 10, k=0,1,2, \ldots, 300$. The known input $u(k)$ is the sinusoidal signal with amplitude less than 0.1 . The exogenous disturbance input $\omega(k)$ is the uniform noise with amplitude less than 0.6. The fault signal $f(k)$ is

$$
f(k)= \begin{cases}-1, & 140 \leq k \leq 250 \\ 0, & \text { others. }\end{cases}
$$

Now, four cases for different transition rate matrix (TRM) are shown in Table 1, and the simulation result of Markov chain $\varsigma(k)$ is given in Figure 2. 
TABLE 1: Four different TRMs.

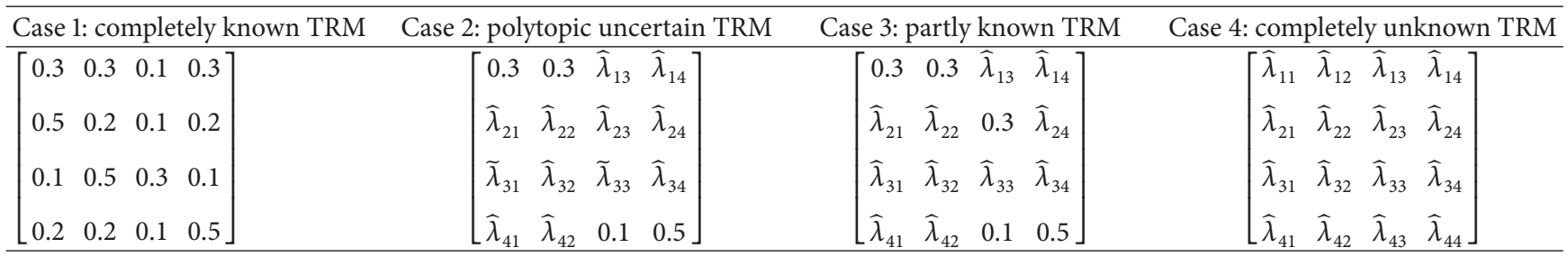

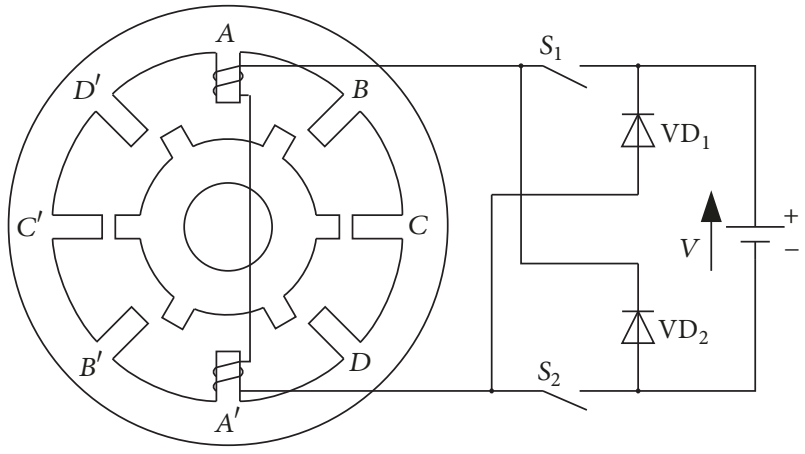

FIGURE 1: Four-phase SRM system structure.

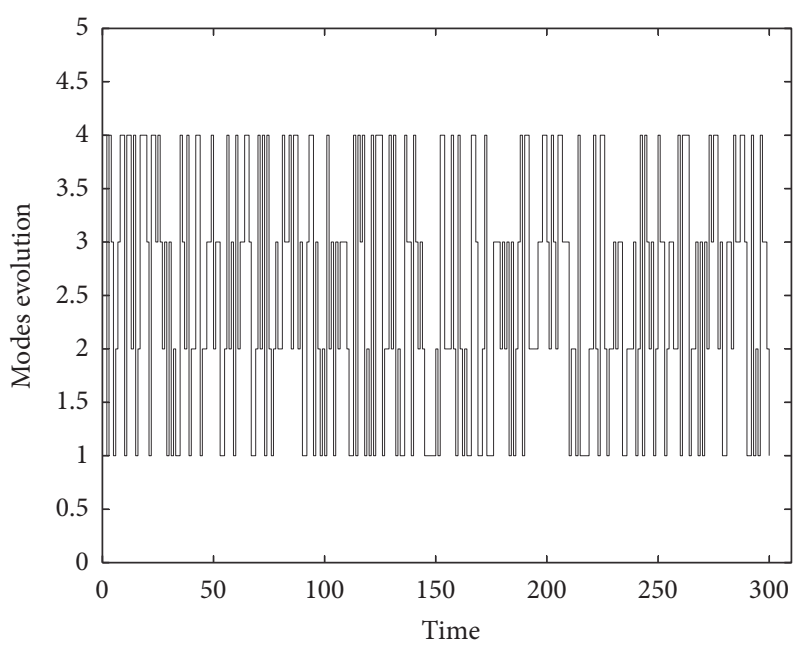

FIgURE 2: Modes evolution of SRM system.

For case 2, the TRM includes two vertices $\Delta_{\zeta}, \varsigma=1,2$, and their third rows $\Delta_{\varsigma}^{3}, \varsigma=1,2$, are given by

$$
\begin{aligned}
\Delta_{1}^{3} & =\left[\begin{array}{llll}
0.6 & \hat{\lambda}_{32} & 0.2 & \hat{\lambda}_{34}
\end{array}\right], \\
\Delta_{2}^{3} & =\left[\begin{array}{llll}
0.42 & \hat{\lambda}_{32} & 0.13 & \hat{\lambda}_{34}
\end{array}\right] .
\end{aligned}
$$

We can obtain the filter gains for four different FD filters with time-varying time delays in Case 2 by applying Theorem 8 and the Matlab LMI Toolbox. The full-order FD filter gains are given as follows:

$$
A_{F 1}=\left[\begin{array}{cc}
0.0538 & -0.2743 \\
0.5513 & 0.3146
\end{array}\right]
$$

$$
\begin{aligned}
& B_{F 1}=\left[\begin{array}{l}
0.5372 \\
0.2574
\end{array}\right] \text {, } \\
& C_{F 1}=\left[\begin{array}{ll}
0.1391 & 0.2793
\end{array}\right], \\
& D_{F 1}=[-0.2931] \text {, } \\
& A_{F 2}=\left[\begin{array}{ll}
0.1201 & 0.2815 \\
0.5213 & 0.1801
\end{array}\right] \text {, } \\
& B_{F 2}=\left[\begin{array}{c}
-0.8387 \\
1.3149
\end{array}\right], \\
& C_{F 2}=\left[\begin{array}{ll}
0.5768 & 0.3691
\end{array}\right] \text {, } \\
& D_{F 2}=[-0.1492] \text {, } \\
& A_{F 3}=\left[\begin{array}{cc}
0.1579 & -0.3801 \\
-0.0513 & -0.0496
\end{array}\right] \text {, } \\
& B_{F 3}=\left[\begin{array}{c}
0.2103 \\
-0.4689
\end{array}\right] \text {, } \\
& C_{F 3}=\left[\begin{array}{ll}
1.4592 & 1.5871
\end{array}\right], \\
& D_{F 3}=[-0.0051] \text {, } \\
& A_{F 4}=\left[\begin{array}{cc}
0.2413 & -0.3869 \\
0.3701 & 0.6578
\end{array}\right] \text {, } \\
& B_{F 4}=\left[\begin{array}{c}
0.9136 \\
-1.3497
\end{array}\right] \text {, } \\
& C_{F 4}=\left[\begin{array}{ll}
1.4103 & 0.2526
\end{array}\right] \text {, } \\
& D_{F 4}=[0.4359] \text {, }
\end{aligned}
$$

and the reduced-order FD filter gains are given as follows:

$$
\begin{aligned}
& \widetilde{A}_{F 1}=[0.1518], \\
& \widetilde{B}_{F 1}=[0.6201], \\
& \widetilde{C}_{F 1}=[0.1435], \\
& \widetilde{D}_{F 1}=[-0.6879], \\
& \widetilde{A}_{F 2}=[0.1179],
\end{aligned}
$$



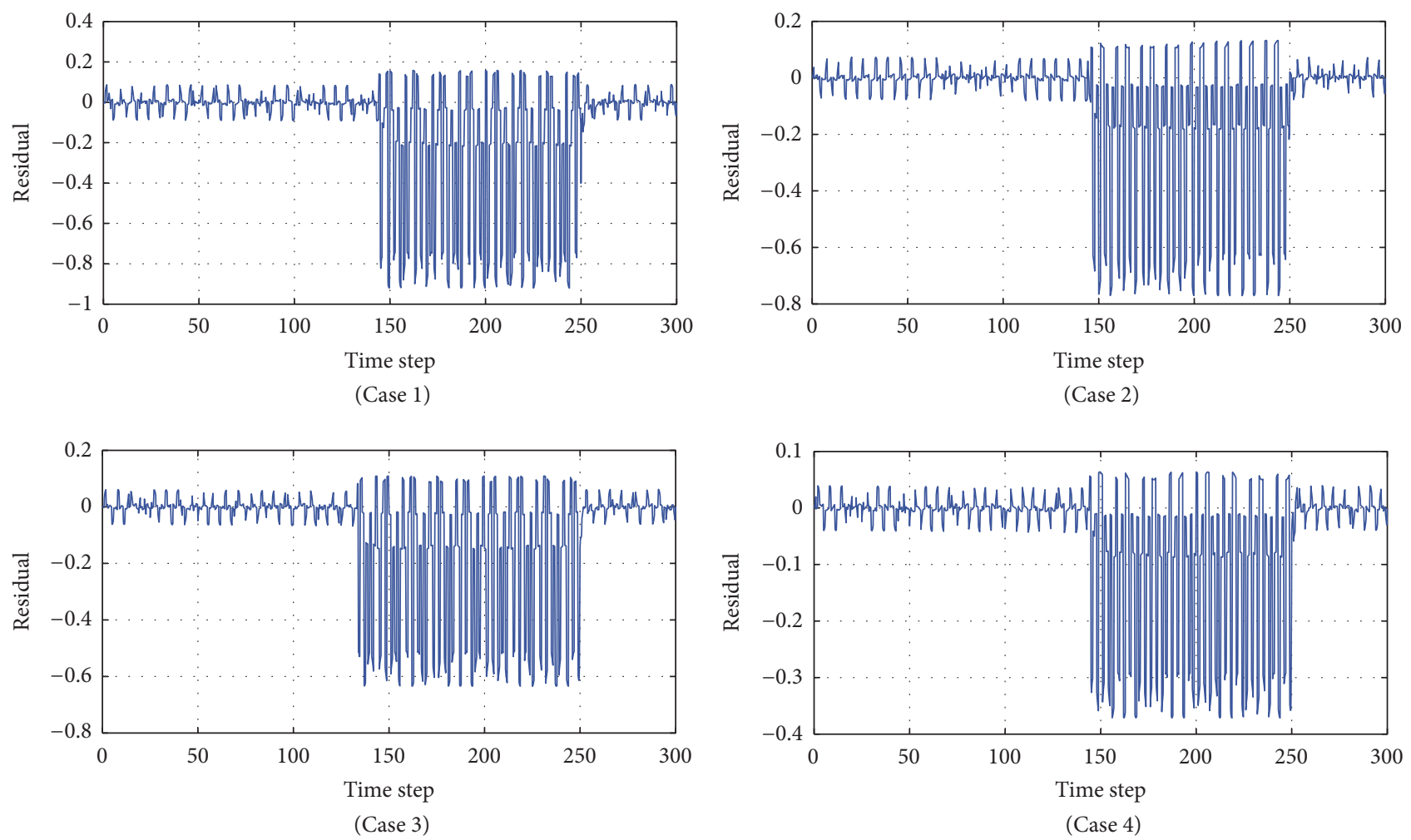

FIgURE 3: Residual generation of full-order fault detection filter.

$$
\begin{aligned}
& \widetilde{B}_{F 2}=[-0.8392], \\
& \widetilde{C}_{F 2}=[0.3681], \\
& \widetilde{D}_{F 2}=[-0.1493], \\
& \widetilde{A}_{F 3}=[0.2094], \\
& \widetilde{B}_{F 3}=[-0.4659], \\
& \widetilde{C}_{F 3}=[1.5791], \\
& \widetilde{D}_{F 3}=[-0.0052], \\
& \widetilde{A}_{F 4}=[0.6512], \\
& \widetilde{B}_{F 4}=[-1.3493], \\
& \widetilde{C}_{F 4}=[-0.7702], \\
& \widetilde{D}_{F 4}=[-0.4413] .
\end{aligned}
$$

The feasible solutions for the other three TRM cases shown in Table 1 are omitted for brevity.

Obviously, it is seen that the residual value estimated by the full-order filter is closer to the weighting fault signal than the estimated residual value of the reduced-order filter from the comparison of Figures 3 and 5. The more the known transition rates are, the more accurate the estimated residual values are. The simulation results further indicate that, whether the transition rates are known or not, the designed fault detection filters are effective and feasible.

Figures 4 and 6 display the evolutions of $J(\widehat{r}(k))=$ $\sqrt{\sum_{k=k_{0}}^{k_{0}+L} \widehat{r}^{T}(k) \widehat{r}(k)}$ for both faulty case and fault-free case, respectively. For different cases, the residuals are different, the calculated residual evaluation functions are different, and the corresponding thresholds $J_{\text {th }} \triangleq \sup _{d \in l_{2}, f=0} E[J(\widehat{r}(k))]$ are different. Weighting fault signal method can improve the performance of the fault detection system.

In Tables 2 and 3, the characteristics of the full-order fault detection filter and the reduced-order fault detection filter are compared in the case of different delay differentials. As the time-varying delay differential value decreases, the time steps to detect the fault are shortened. From the comparison results of the same-order FD filters, it is clear to show that the performance of fault detection filter in polytopic uncertain TRs is superior to those in incompletely known and completely unknown TRs. In this example, we can see that the fault detection time does not change much as the order of fault detection filter is reduced. Thus, it declares the effectiveness of the designed FD reduced-order filter for MJLSs with deficient transition information.

\section{Conclusion}

In this paper, a fault detection filtering method has been investigated for polytopic uncertain discrete-time MJLSs with time-varying delays. The main contribution of our research is the introduction of fault detection reduced-order filter design 


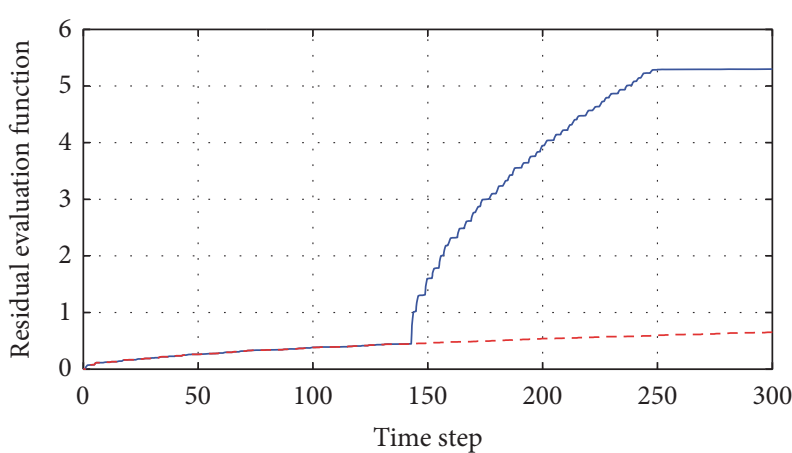

(Case 1)

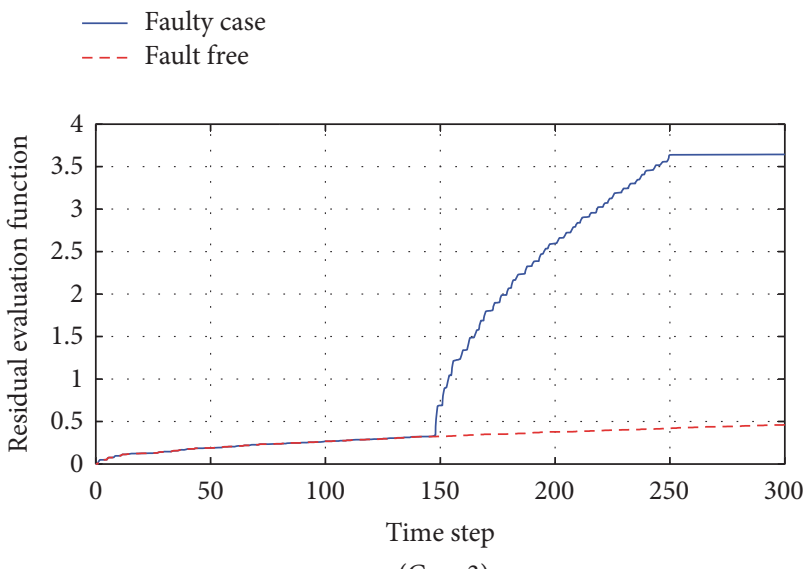

(Case 3)

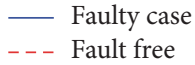

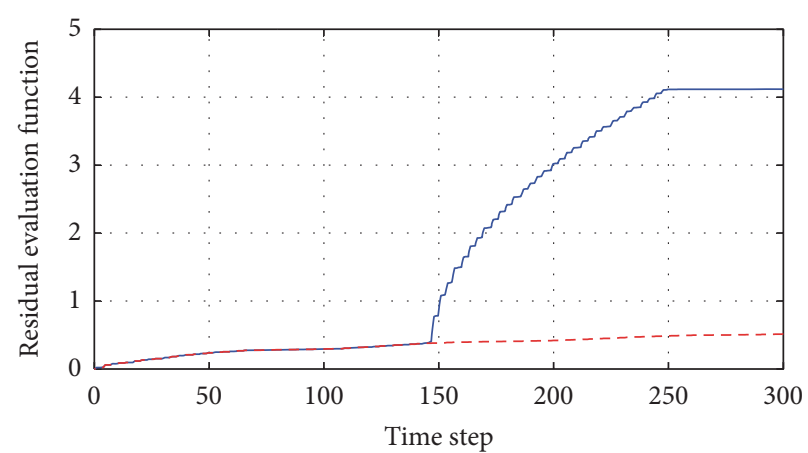

(Case 2)

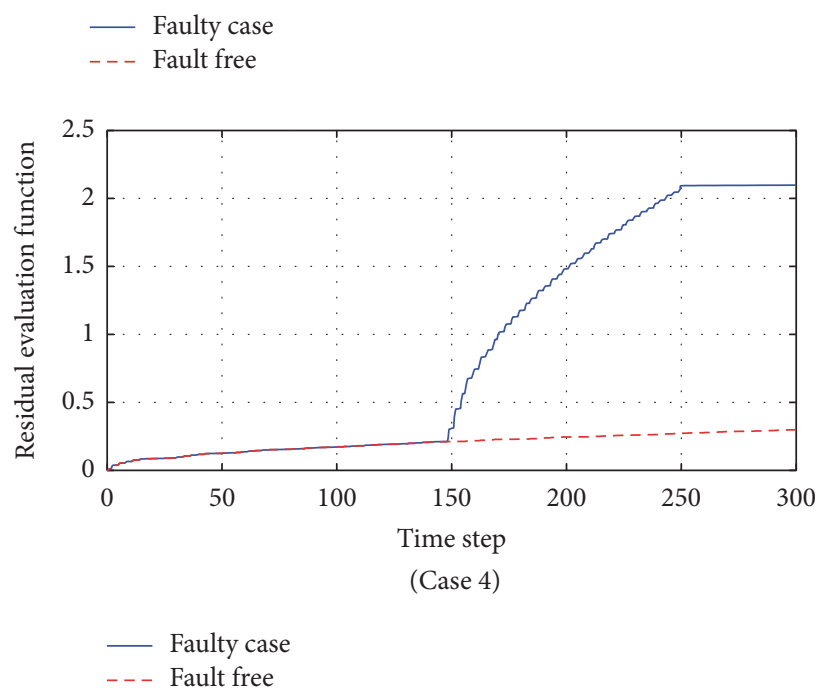

FIGURE 4: Evaluation function curve of full-order fault detection filter.

TABLE 2: Comparison results for four different full-order FD filters cases with time-varying delays.

\begin{tabular}{|c|c|c|c|}
\hline $\begin{array}{l}\text { Transition rate matrix } \\
\text { TRM }\end{array}$ & $\begin{array}{c}\text { Minimum } H_{\infty} \text { performance } \\
d_{12}=2\end{array}$ & $\begin{array}{c}\text { Threshold } J_{\text {th }} \\
d_{12}=2\end{array}$ & $\begin{array}{c}\text { Time steps } \\
d_{12}=2\end{array}$ \\
\hline Case 1 & 1.6715 & 0.4358 & 143 \\
\hline Case 2 & 1.9437 & 0.4167 & 145 \\
\hline Case 3 & 2.0791 & 0.3333 & 146 \\
\hline Case 4 & 2.1932 & 0.2291 & 148 \\
\hline $\begin{array}{l}\text { Transition rate matrix } \\
\text { TRM }\end{array}$ & $\begin{array}{c}\text { Minimum } H_{\infty} \text { performance } \\
d_{12}=6\end{array}$ & $\begin{array}{c}\text { Threshold } J_{\text {th }} \\
d_{12}=6\end{array}$ & $\begin{array}{c}\text { Time steps } \\
d_{12}=6\end{array}$ \\
\hline Case 1 & 3.1742 & 0.4681 & 144 \\
\hline Case 2 & 3.4379 & 0.4462 & 146 \\
\hline Case 3 & 3.6153 & 0.3615 & 147 \\
\hline Case 4 & 3.8256 & 0.2549 & 149 \\
\hline
\end{tabular}

for polytopic uncertain MJLSs with time-varying delays. It should be emphasized that the above-mentioned approach is used in the fault detection process of polytopic uncertain discrete-time MJLSs for the first time. The underlying systems are more common. Based on Wirtinger-based inequality and Markovian Lyapunov functional, some sufficient conditions for the existence of FD reduced-order filter for polytopic uncertain discrete-time MJLSs with time-varying delays are obtained, such that the filtering error system is randomly stable. Finally, an effective example has been adopted to fully illustrate the effectiveness of the proposed design approach, which can improve the sensitivity of fault detection and reduce the fault detection rate of false positives. Further research is anticipated by extending to the fault tolerant control methods for time-varying delay and uncertain process in the different Markov systems. 

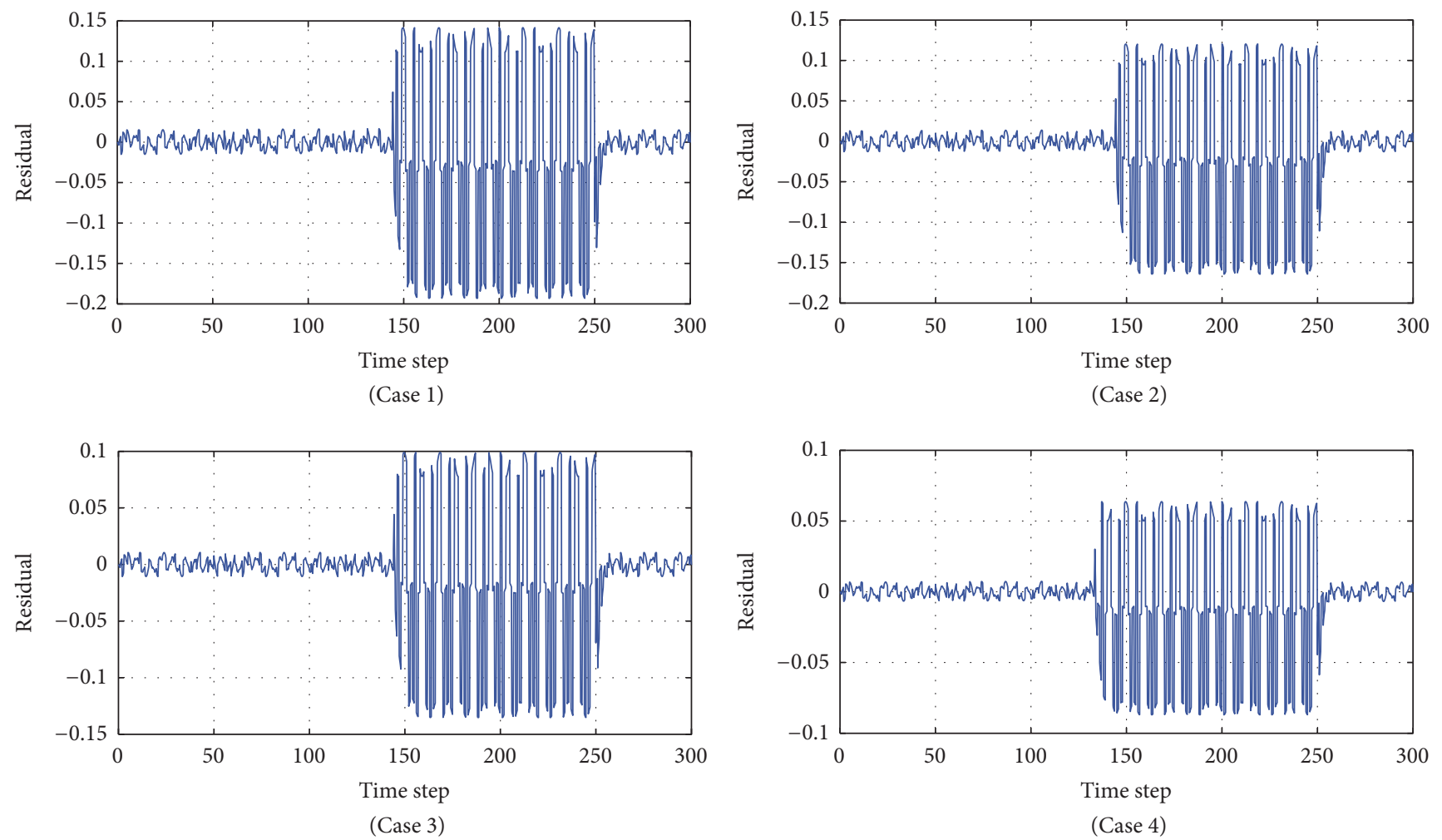

FIGURE 5: Residual generation of reduced-order fault detection filter.

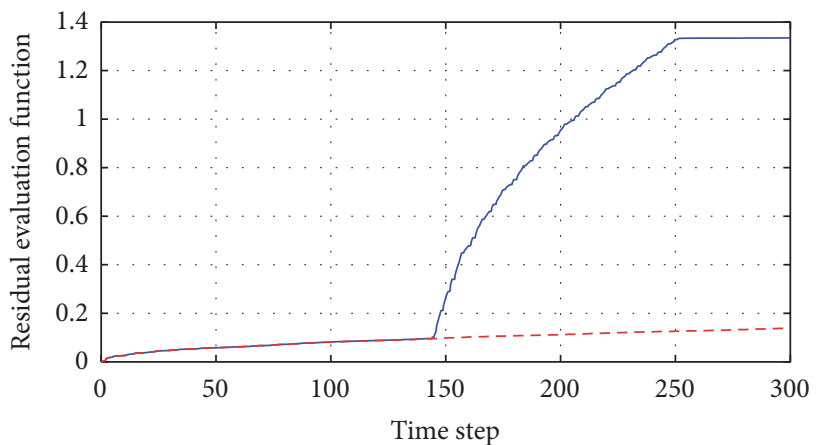

(Case 1)

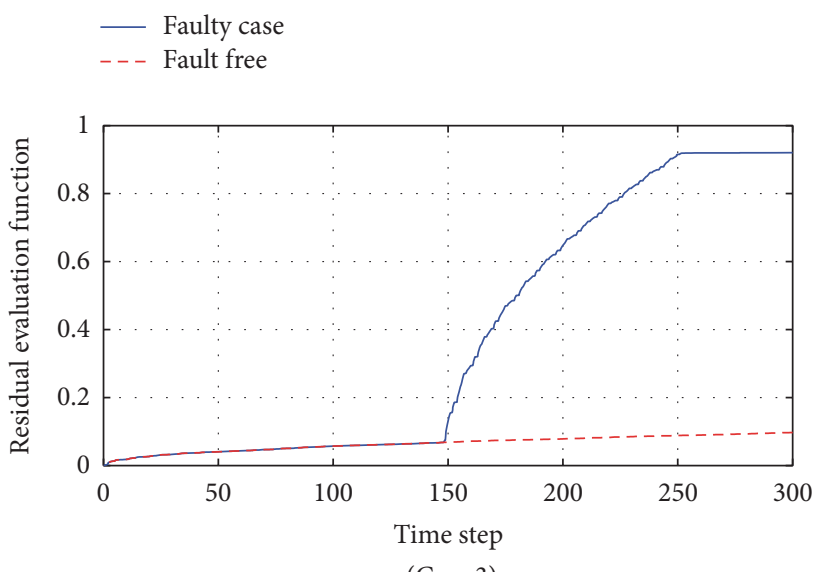

(Case 3)

- Faulty case - - - Fault free

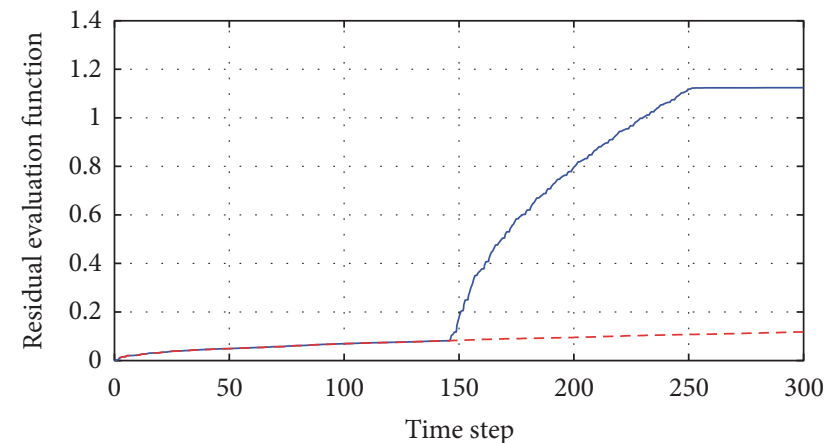

(Case 2)

- Faulty case

- . Fault free

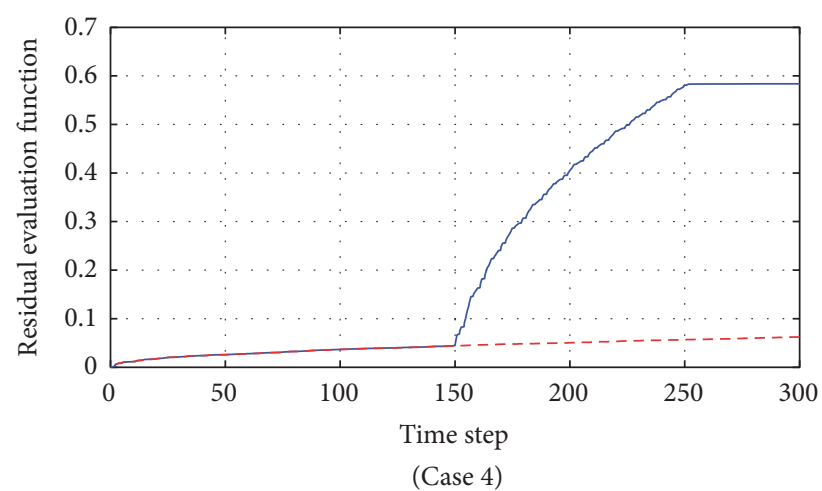

- Faulty case

- - - Fault free

FIGURE 6: Evaluation function curve of reduced-order fault detection filter. 
TABLE 3: Comparison results for four different reduced-order FD filters cases with time-varying delays.

\begin{tabular}{|c|c|c|c|}
\hline $\begin{array}{l}\text { Transition rate matrix } \\
\text { TRM }\end{array}$ & $\begin{array}{c}\text { Minimum } H_{\infty} \text { performance } \\
d_{12}=2\end{array}$ & $\begin{array}{c}\text { Threshold } J_{\text {th }} \\
d_{12}=2\end{array}$ & $\begin{array}{c}\text { Time steps } \\
d_{12}=2\end{array}$ \\
\hline case 1 & 1.7492 & 0.1125 & 144 \\
\hline case 2 & 2.0139 & 0.0875 & 146 \\
\hline case 3 & 2.1521 & 0.0667 & 147 \\
\hline case 4 & 2.2378 & 0.0375 & 149 \\
\hline $\begin{array}{l}\text { Transition rate matrix } \\
\text { TRM }\end{array}$ & $\begin{array}{c}\text { Minimum } H_{\infty} \text { performance } \\
d_{12}=6\end{array}$ & $\begin{array}{c}\text { Threshold } J_{\text {th }} \\
d_{12}=6\end{array}$ & $\begin{array}{c}\text { Time steps } \\
d_{12}=6\end{array}$ \\
\hline case 1 & 3.5146 & 0.1276 & 145 \\
\hline case 2 & 3.7943 & 0.0913 & 147 \\
\hline case 3 & 3.9018 & 0.0741 & 148 \\
\hline case 4 & 4.0157 & 0.0489 & 150 \\
\hline
\end{tabular}

\section{Conflicts of Interest}

The authors declare that there are no conflicts of interest regarding the publication of this paper.

\section{Acknowledgments}

This work was supported by the National Natural Science Foundation of China (61503091) and the Education Science Planning Key Project of Heilongjiang Province (GJB1316017).

\section{References}

[1] E. K. Boukas and Z. K. Liu, "Robust H-infinity control of discrete-time Markovian jump linear systems with modedependent time-delays," IEEE Transactions on Automatic Control, vol. 46, no. 12, pp. 1918-1924, 2001.

[2] Y. Wei, J. Qiu, and S. Fu, "Mode-dependent nonrational output feedback control for continuous-time semi-Markovian jump systems with time-varying delay," Nonlinear Analysis: Hybrid Systems, vol. 16, pp. 52-71, 2015.

[3] Y. Wei, J. H. Park, J. Qiu, L. Wu, and H. Y. Jung, "Sliding mode control for semi-Markovian jump systems via output feedback," Automatica, vol. 81, pp. 133-141, 2017.

[4] A. Ahadi, N. Ghadimi, and D. Mirabbasi, "An analytical methodology for assessment of smart monitoring impact on future electric power distribution system reliability," Complexity, vol. 21, no. 1, pp. 99-113, 2015.

[5] Y. Wei, J. Qiu, H. R. Karimi, and M. Wang, "Model approximation for two-dimensional Markovian jump systems with statedelays and imperfect mode information," Multidimensional Systems and Signal Processing, vol. 26, no. 3, pp. 575-597, 2015.

[6] Y. Wei, J. Qiu, and H. Karimi, "Quantized Ho filtering for continuous-time Markovian jump systems with deficient mode information," Asian Journal of Control, vol. 17, no. 6, pp. 19141923, 2015.

[7] R. Sakthivel and S. Selvi, "Robust reliable sampled-data Hinfinity control for uncertain stochastic systems with random delay," Complexity, vol. 21, no. 2, pp. 42-58, 2015.

[8] Y. Wei, J. Qiu, and H. Karimi, "New results on Ho dynamic output feedback control for Markovian jump systems with time-varying delay and defective mode information," Optimal
Control, Applications and Methods, vol. 35, no. 6, pp. 656-675, 2014.

[9] Y. Wei, J. Qiu, P. Shi, and M. Chadli, "Fixed-Order PiecewiseAffine Output Feedback Controller for Fuzzy-Affine-ModelBased Nonlinear Systems with Time-Varying Delay,' IEEE Transactions on Circuits and Systems I: Regular Papers, vol. 64, no. 4, pp. 945-958, 2017.

[10] Y. Wei, J. Qiu, and H. Karimi, "Ho model reduction for continuous-time Markovian jump systems with incomplete statistics of mode information," International Journal of Systems Science, vol. 45, no. 7, pp. 1496-1507, 2014.

[11] M. Mohammadi, A. Danandeh, H. Nasir Aghdam, and N. Ojaroudi, "Wavelet neural network based on islanding detection via inverter-based DG," Complexity, vol. 21, no. 2, pp. 309324, 2015.

[12] M. Zhong, H. Ye, and P. Shi, "Fault detection for Markovian jump systems," IEE Proceedings Control Theory and Applications, vol. 152, no. 4, pp. 397-402, 2005.

[13] Y. Wei, J. Qiu, and H. R. Karimi, "Fuzzy-affine-model-based memory filter design of nonlinear systems with time-varying delay," IEEE Transactions on Fuzzy Systems, vol. PP, no. 99, March 2017.

[14] A. Mishra and A. K. Tripathi, "Complexity of a problem of energy efficient real-time task scheduling on a muticore processor," Complexity, vol. 21, no. 1, pp. 259-267, 2015.

[15] K. Mathiyalagan, J. H. Park, and R. Sakthivel, “Observer-based dissipative control for networked control systems: a switched system approach," Complexity, vol. 21, no. 2, pp. 297-308, 2015.

[16] M. Faraji-Niri, M. R. Jahed-Motlagh, and M. BarkhordariYazdi, "Stabilization of active fault-tolerant control systems by uncertain nonhomogeneous Markovian jump models," Complexity, vol. 21, no. S1, pp. 318-329, 2016.

[17] Y. Wei, J. Qiu, and H. R. Karimi, "Reliable output feedback control of discrete-time fuzzy affine systems with actuator faults," IEEE Transactions on Circuits Systems-I Regular Papers, vol. 64, no. 1, pp. 170-181, 2017.

[18] Y. Wei, J. Qiu, H. K. Lam, and L. Wu, "Approaches to T-S fuzzy-affine-model-based reliable output feedback control for nonlinear Ito stochastic systems," IEEE Transactions on Fuzzy Systems, vol. 25, no. 3, pp. 569-583, 2017.

[19] X. Wan, H. Fang, and F. Yang, "Fault detection for a class of networked nonlinear systems subject to imperfect measurements," International Journal of Control, Automation, and Systems, vol. 10, no. 2, pp. 265-274, 2012. 
[20] Y. Wei, J. H. Park, H. R. Karimi, Y.-C. Tian, and H. Jung, "Improved Stability and Stabilization Results for Stochastic Synchronization of Continuous-Time Semi-Markovian Jump Neural Networks With Time-Varying Delay," IEEE Transactions on Neural Networks and Learning Systems, 2017.

[21] Y. Wei, J. Qiu, and H. K. Lam, "A novel approach to reliable output feedback control of fuzzy-affine systems with timedelays and sensor faults," IEEE Transactions on Fuzzy Systems, vol. 25, no. 6, pp. 1808-1823, 2017.

[22] L. Lavigne, A. Zolghadri, P. Goupil, and P. Simon, "A modelbased technique for early and robust detection of oscillatory failure case in A380 actuators," International Journal of Control, Automation, and Systems, vol. 9, no. 1, pp. 42-49, 2011.

[23] H. Hamdi, M. Rodrigues, C. Mechmeche, and N. B. Braiek, "Robust fault detection and estimation for descriptor systems based on multi-models concept," International Journal of Control, Automation, and Systems, vol. 10, no. 6, pp. 1260-1266, 2012.

[24] Y. Wei, J. H. Park, J. Qiu, and H. Jung, "Reliable Output Feedback Control for Piecewise Affine Systems with MarkovType Sensor Failure," IEEE Transactions on Circuits and Systems II: Express Briefs, 2017.

[25] S. K. Nguang, P. Shi, and S. Ding, "Fault detection for uncertain fuzzy systems: An LMI approach," IEEE Transactions on Fuzzy Systems, vol. 15, no. 6, pp. 1251-1262, 2007.

[26] Y. Wei, J. Qiu, P. Shi, and L. Wu, "A piecewise-markovian lyapunov approach to reliable output feedback control for fuzzy-affine systems with time-delays and actuator faults," IEEE Transactions on Cybernetics, vol. PP, no. 99, pp. 1-13, 2017.

[27] P. Park, J. W. Ko, and C. Jeong, "Reciprocally convex approach to stability of systems with time-varying delays," Automatica, vol. 47, no. 1, pp. 235-238, 2011.

[28] Y. Wei, J. Qiu, H. R. Karimi, and W. Ji, "A Novel Memory Filtering Design for Semi-Markovian Jump Time-Delay Systems," IEEE Transactions on Systems, Man, and Cybernetics: Systems, vol. PP, no. 99, pp. 1-13, 2017.

[29] A. Seuret, F. Gouaisbaut, and E. Fridman, "Stability of discretetime systems with time-varying delays via a novel summation inequality," Institute of Electrical and Electronics Engineers Transactions on Automatic Control, vol. 60, no. 10, pp. 27402745, 2015.

[30] Y. Wei, J. Qiu, P. Shi, and H.-K. Lam, "A new design of H-infinity piecewise filtering for discrete-time nonlinear time-varying delay systems via T-S fuzzy affine models," IEEE Transactions on Systems, Man, and Cybernetics: Systems, vol. 47, no. 8, pp. 20342047, 2017.

[31] H. Yang, B. Jiang, and V. Cocquempot, "A fault tolerant control framework for periodic switched non-linear systems," International Journal of Control, vol. 82, no. 1, pp. 117-129, 2009. 


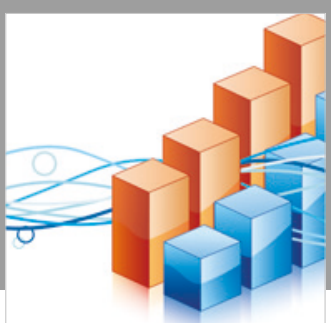

Advances in

Operations Research

\section{-n-m}
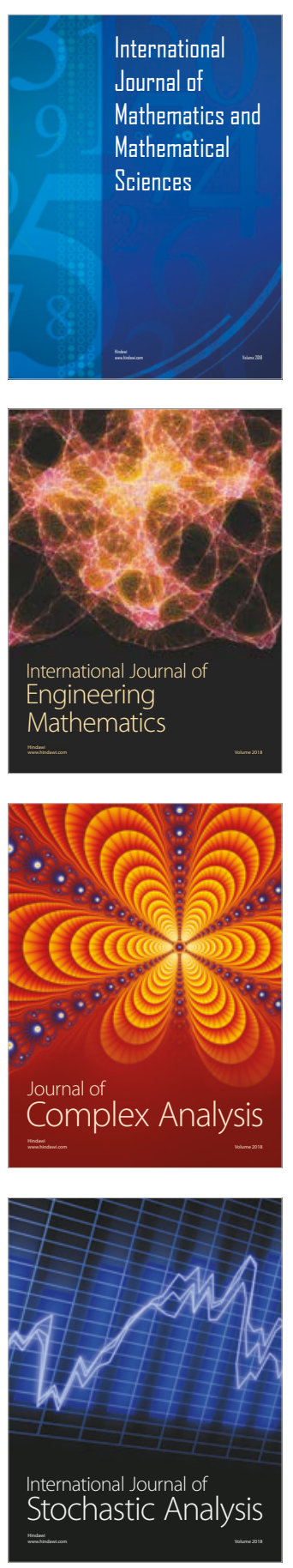
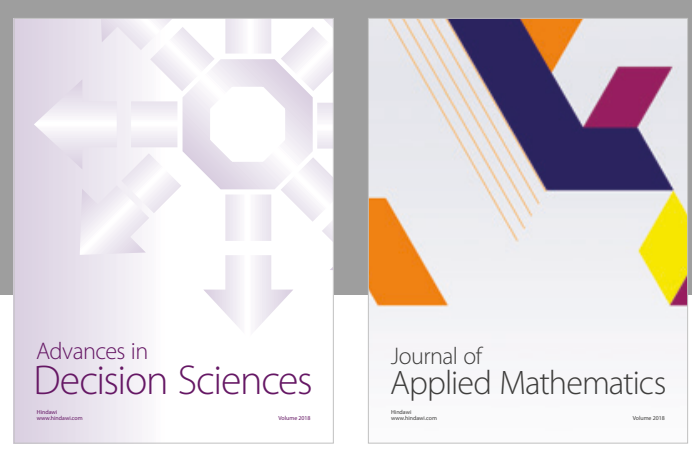

Journal of

Applied Mathematics
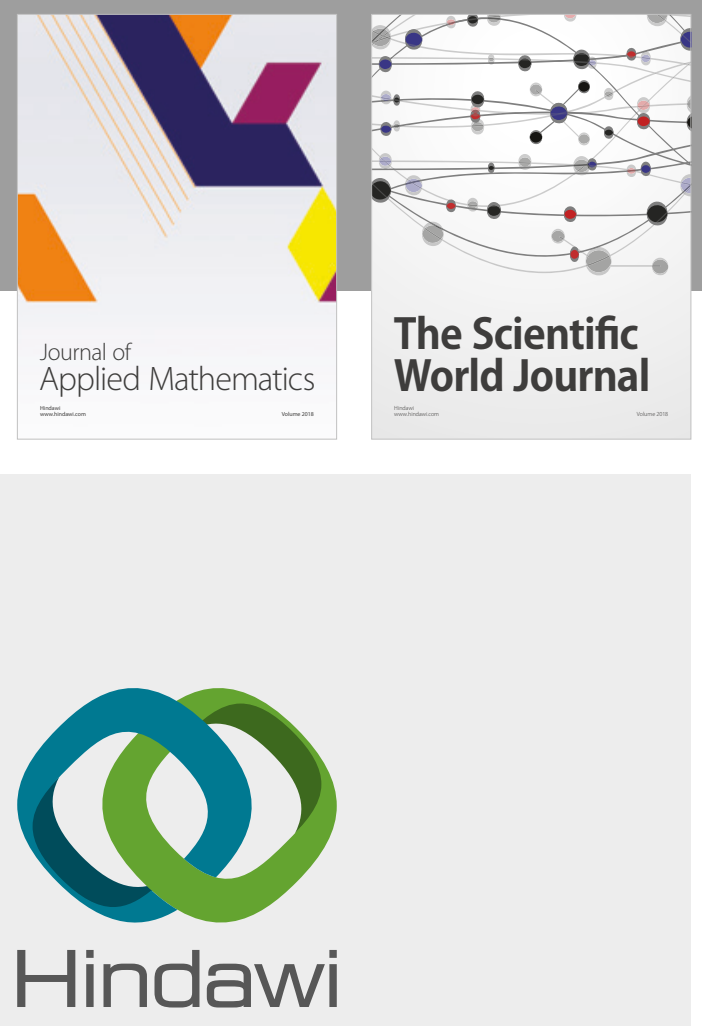

Submit your manuscripts at

www.hindawi.com

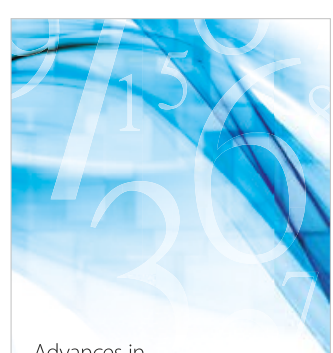

Advances in
Numerical Analysis
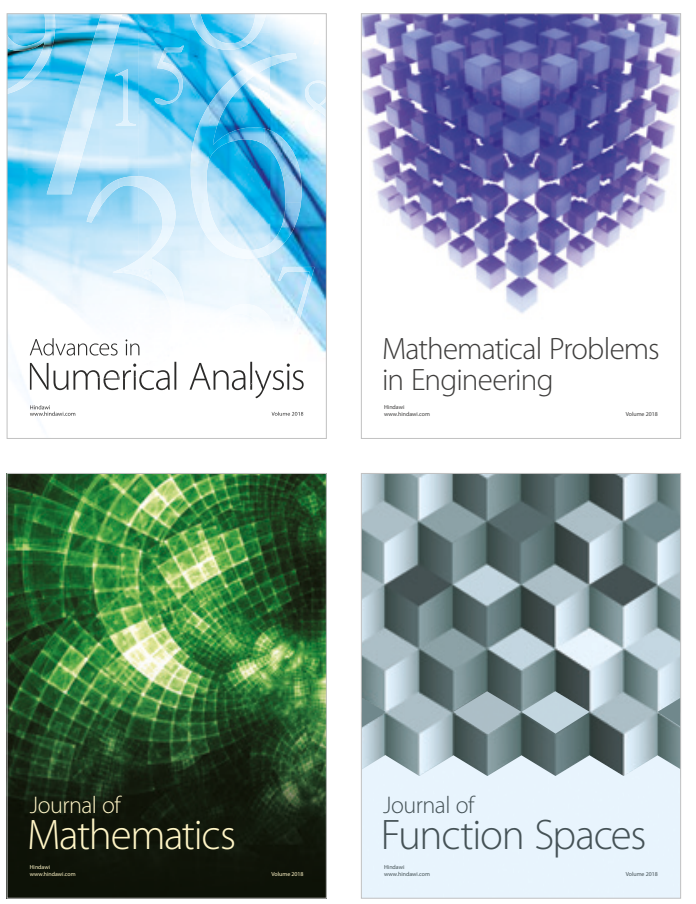

Mathematical Problems in Engineering

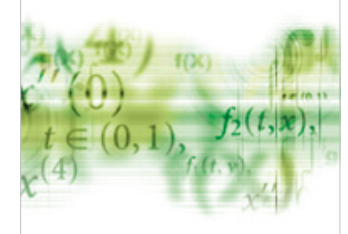

International Journal of

Differential Equations

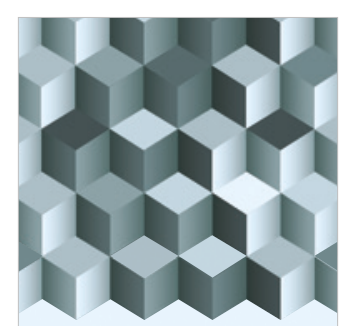

Journal of

Function Spaces
The Scientific

World Journal

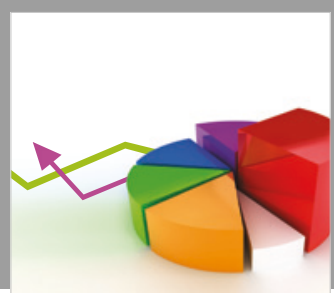

Journal of

Probability and Statistics
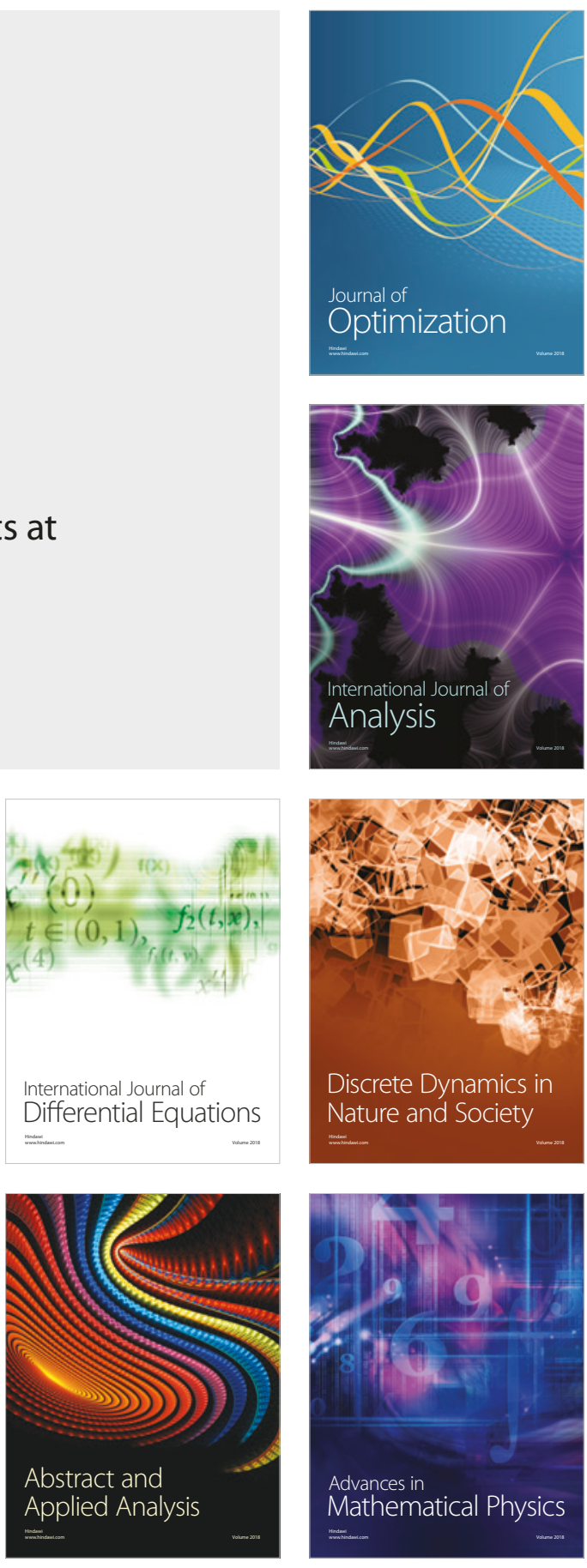